\title{
Subunit-Specific Photocontrol of Glycine Receptors by Azobenzene-Nitrazepam Photoswitcher
}

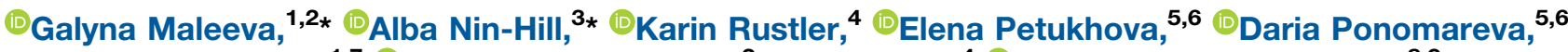 \\ Elvira Mukhametova, ${ }^{1,7}$ (D) Alexandre MJ Gomila, ${ }^{2}$ Daniel Wutz, ${ }^{4}{ }^{\circ}$ Mercedes Alfonso-Prieto, ${ }^{8,9}$ \\ Birkhard König, ${ }^{4}$ (D)Pau Gorostiza, ${ }^{2,10,11}$ and ${ }^{\circledR P}$ Piotr Bregestovski ${ }^{1,5,6}$
}

\section{https://doi.org/10.1523/ENEURO.0294-20.2020}

${ }^{1}$ Institut National de la Santé et de la Recherche Médicale, Institut de Neurosciences des Systèmes, Aix-Marseille University, Marseille 13005, France, ${ }^{2}$ Institute for Bioengineering of Catalonia, The Barcelona Institute of Science and Technology, Barcelona 08028, Spain, ${ }^{3}$ Department of Inorganic and Organic Chemistry (Section of Organic Chemistry) and Institute of Theoretical Chemistry (IQTCUB), University of Barcelona, Barcelona 08028, Spain, ${ }^{4}$ Institute of Organic Chemistry, University of Regensburg, Regensburg 93053, Germany, ${ }^{5}$ Department of Normal Physiology, Kazan State Medical University, Kazan 420012, Russia, ${ }^{6}$ Institute of Neurosciences, Kazan State Medical University, Kazan 420012, Russia, ${ }^{7}$ Open Lab of Motor Neurorehabilitation, Kazan Federal University, Kazan 420008, Russia, 8 Institute for Advanced Simulation IAS-5 and Institute of Neuroscience and Medicine INM-9, Computational Biomedicine, Forschungszentrum Jülich, Jülich 52425, Germany, ${ }^{9}$ Cécile and Oskar Vogt Institute for Brain Research, Medical Faculty, Heinrich Heine University Düsseldorf, Düsseldorf 40225, Germany, ${ }^{10}$ Catalan Institution for Research and Advanced Studies, Barcelona 08003, Spain, and ${ }^{11}$ Centro de Investigación Biomédica en Red en Bioingeniería, Biomateriales y Nanomedicina, Madrid 28001, Spain

\section{Visual Abstract}
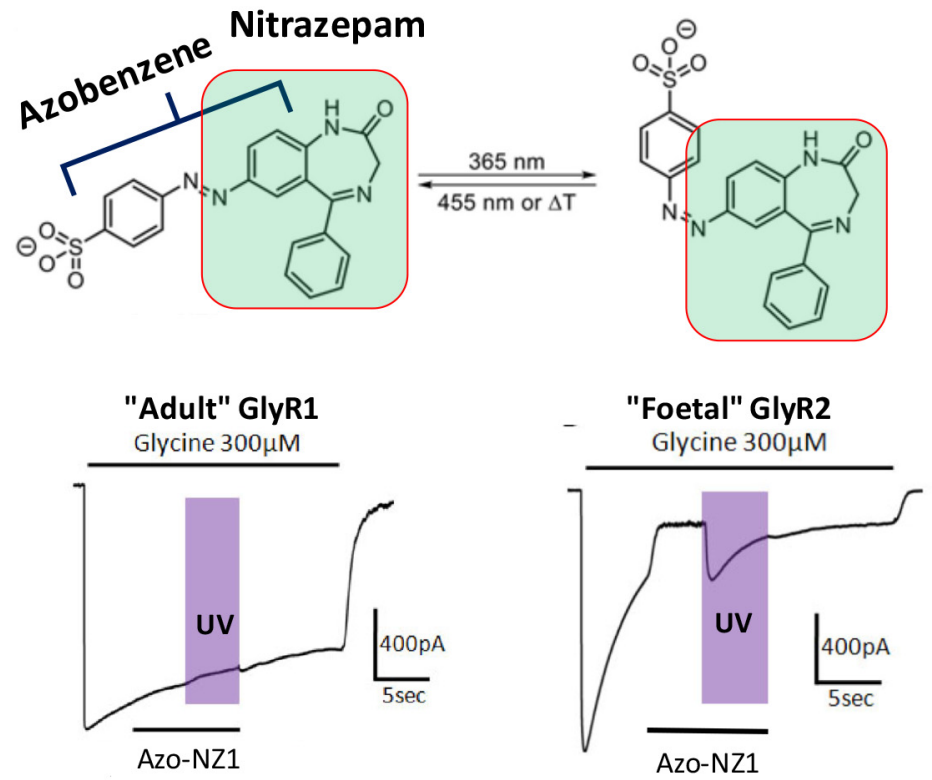

$$
\text { "Adult" GlyR1 PARVGLGITTVLTMTTQSSGS }
$$$$
\text { "Foetal" GlyR2 PARVALGITTVLTMTTQSSGS }
$$

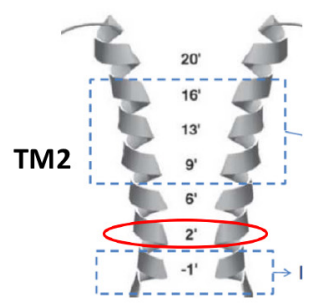

Synaptic currents

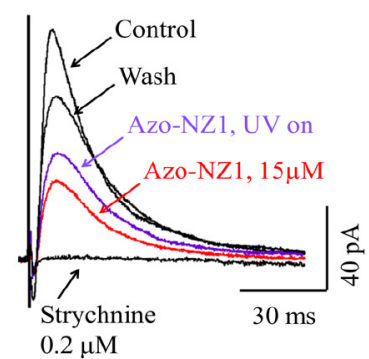

Photopharmacology is a unique approach that through a combination of photochemistry methods and advanced life science techniques allows the study and control of specific biological processes, ranging from intracellular pathways to brain circuits. Recently, a first photochromic channel blocker of anion-selective $G_{A B A}$ receptors, the azobenzene-nitrazepam-based photochromic compound (Azo-NZ1), has been described. In the present study, using patch-clamp technique in heterologous system and in mice brain slices, site-directed 


\section{Significance Statement}

Photochromic molecules are becoming widely used for studying and modulating various biological processes. Successful application of these compounds, whose activity can be controlled with light, potentially provides a promising tool for future therapeutic approaches. The main advantage of such compounds is their precise spatial and temporal selectivity, a property that favors specific drug action and diminishes their side effects. In the present study, we describe in detail the interaction of the novel azobenzene-nitrazepambased photochromic compound (Azo-NZ1) with glycine receptors (GlyRs) and determine its subunit-specific blocking activity in the $\mathrm{Cl}$-selective pore of GlyRs. This compound offers a new strategy for specific control of glycinergic circuits and stepping stone for design of new GlyR-active drugs.

mutagenesis and molecular modeling we provide evidence of the interaction of Azo-NZ1 with glycine receptors (GlyRs) and determine the molecular basis of this interaction. Glycinergic synaptic neurotransmission determines an important inhibitory drive in the vertebrate nervous system and plays a crucial role in the control of neuronal circuits in the spinal cord and brain stem. GlyRs are involved in locomotion, pain sensation, breathing, and auditory function, as well as in the development of such disorders as hyperekplexia, epilepsy, and autism. Here, we demonstrate that Azo-NZ1 blocks in a UV-dependent manner the activity of $\alpha 2$ GlyRs (GlyR2), while being barely active on $\alpha 1$ GlyRs (GlyR1). The site of Azo-NZ1 action is in the chloride-selective pore of GlyR at the 2' position of transmembrane helix 2 and amino acids forming this site determine the difference in Azo-NZ1 blocking activity between GlyR2 and GlyR1. This subunit-specific modulation is also shown on motoneurons of brainstem slices from neonatal mice that switch during development from expressing "fetal" GlyR2 to "adult" GlyR1 receptors.

Key words: brain slices; glycine receptors; hypoglossal motoneurons; molecular modelling; patch-clamp; photopharmacology

Received July 3, 2020; accepted November 23, 2020; First published December 9, 2020.

The authors declare no competing financial interests.

Author contributions: M.A.-P., B.K., P.G., and P.B. designed research; G.M., A.N.-H., K.R., E.P., D.P., E.M., A.M.J.G., D.W., M.A.-P., and P.B. performed research; K.R. contributed unpublished reagents/analytic tools; G.M., A.N.-H., E.P., D.P., E.M., and P.B. analyzed data; G.M., E.P., M.A.-P., B.K., P.G., and P.B. wrote the paper.

This work was supported by the Russian Science Foundation Grant 18-1500313 (to E.P., D.P., and P.B.), the European Research Area (ERA) SynBIO MODULIGHTOR Grant PCIN-2015-163-C02-01, the Generalitat de Catalunya FIAGAUR PhD Scholarship (A.N.-H.), and an Institute for Bioengineering of Catalonia (IBEC)-BEST Marie Curie Severo Ochoa Postdoctoral Scholarship (G.M.). A.M.J.G. was supported by fellowship BES-2017-083025. Computer resources were provided by MareNostrum IV and MinoTauro and technical support by Barcelona Supercomputing Center, Grant BCV-2017-2-0004. This research received funding from the European Union Research and Innovation Programme Horizon 2020 Human Brain Project SG3 (945539), DEEPER (ICT-36-2020-101016787), Agency for Management of University and Research Grants/Generalitat de Catalunya (CERCA Programme; 2017-SGR-1442 project), Fonds Européen de Développement Économique et Régional (FEDER) funds, Ministry of Science and Innovation (Grant PID2019-111493RB- I00), "la Caixa" foundation (ID 100010434, agreementLCF/PR/ HR19/52160010). The project Clúster Emergent del Cervell Humà (CECH, 001-P001682) is co-financed by the European Union Regional Development Fund within the framework of the ERDF Operational Program of Catalonia 2014-2020 with a grant of $50 \%$ of total eligible cost.

${ }^{*} \mathrm{G} . \mathrm{M}$. and A.N.-H. contributed equally to this work

Acknowledgements: We thank Yuri Zilberter for valuable comments.

Correspondence should be addressed to Galyna Maleeva at gmalieieva@ ibecbarcelona.eu or Mercedes Alfonso-Prieto at malfonsoprieto@gmail.com or Piotr Bregestovski at pbreges@gmail.com.

https://doi.org/10.1523/ENEURO.0294-20.2020 Copyright (C) 2021 Maleeva et al.

This is an open-access article distributed under the terms of the Creative Commons Attribution 4.0 International license, which permits unrestricted use, distribution and reproduction in any medium provided that the original work is properly attributed.

\section{Introduction}

Photopharmacology, a rapidly developing field of research, provides a unique strategy for precise light-driven control of the activity of biological molecules (Broichhagen et al., 2015). Several classes of photochromic compounds have already been successfully used to selectively modulate the function of various proteins, including enzymes (Harvey and Abell, 2001), synaptic receptors, and voltage-gated channels (Banghart et al., 2004, 2009; Gorostiza and Isacoff, 2007, 2008; Tochitsky et al., 2012; Fehrentz et al., 2018). A number of photochromic agonists and antagonists are available for specific control of ionotropic excitatory kainate, AMPA or NMDA receptors (Volgraf et al., 2006; Kienzler et al., 2013; Berlin et al., 2016), as well as nicotinic acetylcholine receptor (Bartels et al., 1971; Lester et al., 1979; Tochitsky et al., 2012). Several optically switchable modulators of inhibitory $\mathrm{GABA}_{\mathrm{A}}$ receptors were developed as well (Lin et al., 2015; Huckvale et al., 2016).

It has been recently shown that azobenzene-nitrazepambased photochromic compound (Azo-NZ1), the azobenzene derivative of nitrazepam, blocks in a UV-dependent manner the ion channel pore of $\mathrm{GABA}_{\mathrm{A}} \alpha 1 / \beta 2 / \gamma 2$ and $\mathrm{GABA}_{\mathrm{C}}$ rho2 receptors and controls GABAergic currents (Maleeva et al., 2019). These experiments also suggested that Azo-NZ1 is capable to modulate the activity of another subtype of Cysloop receptors, $\alpha 2$ glycine receptor (GlyR). In the present study, we investigate in detail the profile of Azo-NZ1 action on different subtypes of GlyRs.

GlyRs are ligand-gated anion-permeable ion channels that belong to the Cys-loop receptor superfamily and provide the main inhibitory drive in the brain stem and spinal cord of vertebrates, as well as regulate network 
excitability in the retina, hippocampus and amygdala (Zarbin et al., 1981; McCool and Botting, 2000; Chattipakorn and McMahon, 2002; Haverkamp et al., 2003). GlyRs participate in movement control, breathing and processing of sensory information (Schmid et al., 1991; Sassoe-Pognetto et al., 1994; Harvey et al., 2004).

GlyRs represent an important drug target as they are involved in disorders such as hyperekplexia, inflammatory pain sensitization, autism, and temporal lobe epilepsy (Lynch et al., 2017; Zeilhofer et al., 2018). Strychnine is a competitive antagonist of all GlyRs subtypes and is commonly used in electrophysiological experiments to identify glycinergic IPSCs (Young and Snyder, 1973). Numerous studies have shown that strychnine and glycine binding pockets greatly overlap (Vandenberg et al., 1992; Vafa et al., 1999; Du et al., 2015). Several other compounds modulate the activity of GlyRs with strong or partial subunit selectivity. Bicuculline, a classical GABA receptor antagonist, is capable, although with lower efficacy, to also inhibit GlyRs (Fucile et al., 1999). Interestingly, it is much more effective at inhibiting GlyR $\alpha 2$ than GlyR $\alpha 1$ and hence has even been suggested to use for separation of GlyR subtypes (Wang and Slaughter, 2005). Compounds acting on GlyRs as ion channel blockers, also exhibited subunit selectivity. Cyanotriphenylborate (CTB; Rundström et al., 1994) and ginkgolide B, extracted from Ginkgo biloba leaves (Kondratskaya et al., 2005), have been shown as more potent blockers of GlyR $\alpha 1$ compared with GlyR $\alpha 2$, while niflumic acid (NFA), a member of the fenamate class of nonsteroidal anti-inflammatory drugs, on the contrary, was more potent at blocking GlyR $\alpha 2$ s (Maleeva et al., 2017).

Sensitivity of GlyRs to all channel blockers strongly depended on the amino acid located at the 2' position of the ion channel pore. Alkaloids belonging to the picrotoxin (PTX) and ginkgolide groups have more complicate patterns of interaction with GlyRs of different subunit composition, but they have in common a strong dependence on the $\beta$ subunit presence (Pribilla et al., 1992; Kondratskaya et al., 2005; Hawthorne et al., 2006). Such a prominent subtype-determined modulation of GlyRs is promising feature for the development of subunit-specific modulators of GlyRs that will allow a precise control of glycinergic system.

Here, we present a detailed characterization of different GlyRs subtypes interacting with Azo-NZ1, the first photoswitchable ion channel blocker of gamma-aminobutyric acid receptors (GABARs) and GlyRs. We have demonstrated that Azo-NZ1 inhibits GlyRs in a subunit specific manner, being a potent blocker of $\alpha 2$ GlyRs in the transconformation, while nearly inactive in cis-form. Using mutagenesis analysis and molecular modeling, we have shown that the amino acid at 2' position of the ion channel pore is crucial for Azo-NZ1 blocking action.

\section{Materials and Methods}

\section{Chemistry}

Synthesis and photochemical characterization of AzoNZ1 were performed as previously reported (Maleeva et al., 2019).

\section{Cell culture and transfection}

GlyRs of different subunit composition were heterologously expressed in cultured Chinese hamster ovary (CHO) cells obtained from the American type Tissue Culture Collection (ATCC) that were maintained as previously described (Mukhtarov et al., 2013; Maleeva et al., 2015). Transfection was performed using the Lipofectamine 3000 protocol (Life Technology). Cells were transfected with cDNA of the following GlyR subunits: $\alpha 1$ zebrafish, $\alpha 2$ zebrafish, $\alpha 1$ human, $\alpha 1$ human G254A, $\alpha 1$ human $/ \beta$ mouse, $\alpha 2$ mouse/ $\beta$ mouse (hereafter $\alpha 1 \mathrm{Z}, \alpha 2 \mathrm{Z}, \alpha 1 \mathrm{H}, \alpha 1 \mathrm{H}$ G254A, $\alpha 1 \mathrm{H} / \beta \mathrm{M}$, and $\alpha 2 \mathrm{M} / \beta \mathrm{M}$, respectively). For identification of transfected cells, a cDNA of green fluorescent protein (GFP) was co-transfected with cDNA of GlyRs. Three hours after the initial exposure of cells to the cDNAs, the culture medium was replaced with fresh medium containing strychnine $(1 \mu \mathrm{M})$, to reduce the detrimental alterations of membrane potential because of activation of overexpressed GlyRs by glycine present in culture medium. Electrophysiological recordings were conducted on fluorescent cells 24-72 h after transfection.

\section{Electrophysiological recordings on $\mathrm{CHO}$ cells}

Whole-cell patch-clamp recordings were held at room temperature $\left(20-25^{\circ} \mathrm{C}\right)$ using an EPC-9 amplifier (HEKA Elektronik). Cells were continuously superfused with external solution containing the following: $140 \mathrm{~mm} \mathrm{NaCl}, 2$ $\mathrm{mm} \mathrm{CaCl}$, $2.8 \mathrm{~mm} \mathrm{KCl}, 4 \mathrm{~mm} \mathrm{MgCl}_{2}, 20 \mathrm{~mm}$ HEPES, and $10 \mathrm{~mm}$ glucose ( $\mathrm{pH} 7.4,320-330 \mathrm{mOsm})$. Intracellular solution used for filling recording patch pipettes contained the following: $140 \mathrm{~mm} \mathrm{KCl,} 2 \mathrm{~mm} \mathrm{MgCl}$, $2 \mathrm{~mm} \mathrm{MgATP}$, and 2 mм BAPTA (tetrapotassium salt) $(\mathrm{pH} \mathrm{7.3,} 290$ mOsm). Recording pipettes were pulled from borosilicate glass capillaries (Harvard Apparatus Ltd) and had resistances of 5-10 M $\Omega$. Rapid replacement of solutions was provided by fast application system (SF 77A Perfusion Fast-Step, Warner), placed $40-50 \mu \mathrm{m}$ above the recorded cell. Cells with low input resistance $(<150 \mathrm{M} \Omega)$ and a rapid run-down $(>30 \%$ with repetitive application) were excluded from analysis.

Single-channel recordings were performed in the outside-out configuration, at room temperature using an EPC-9 amplifier (HEKA Elektronik). External and internal solutions were the same as those used for whole-cell recordings.

\section{Electrophysiological recordings on brain slices}

Experiments were performed on white laboratory ICR outbred mice of both sexes at postnatal day (P)4-P8. Use of animals was conducted in accordance with the Guide for the Care and Use of Laboratory Animals (NIH Publication No. 85-23, revised 1996) and European Convention for the Protection of Vertebrate Animals used for Experimental and other Scientific Purposes (Council of Europe No. 123; 1985). All animal protocols and experimental procedures were approved by the Local Ethics Committee of Kazan State Medical University (No. 10; 20.12.2016). Mice had free access to food and water and were kept under natural day length fluctuations. 
For electrophysiological experiments, coronal slices containing hypoglossal nucleus were obtained. Mice were decapitated; the brainstems were removed and sliced into 350- $\mu \mathrm{m}$-thick sections using a tissue slicer (model NVSLM1, World Precision Instruments). Sections were prepared in an ice-cold high $\mathrm{K}^{+}$solution, containing the following: $120 \mathrm{~mm}$ K-gluconate, $10 \mathrm{~mm}$ HEPES-acid, 15 mм Na-gluconate, 0.2 mm EGTA, and 4 mм NaCl (pH 7.2, 290-300 mOsm). After cutting, slices were placed for $10 \mathrm{~min}$ at room temperature in a choline-based solution, containing the following: $110 \mathrm{~mm}$ choline chloride, $2.5 \mathrm{~mm} \mathrm{KCl,} 1.25 \mathrm{~mm}$ $\mathrm{NaH}_{2} \mathrm{PO}_{4}, 10 \mathrm{~mm} \mathrm{MgCl}_{2}, 0.5 \mathrm{~mm} \mathrm{CaCl}_{2}, 25 \mathrm{~mm} \mathrm{NaHCO}_{3}, 10$ $\mathrm{mm}$ glucose, and $5 \mathrm{~mm}$ sodium pyruvate $(\mathrm{pH} 7.3-7.4,290-$ $300 \mathrm{mOsm}$ ). Before experiments slices were incubated for 1 $\mathrm{h}$ in a chamber filled with an oxygenated artificial CSF (aCSF) containing the following: $126 \mathrm{~mm} \mathrm{NaCl}, 3.5 \mathrm{~mm} \mathrm{KCl}, 2 \mathrm{~mm}$ $\mathrm{CaCl}_{2}, 1.3 \mathrm{~mm} \mathrm{MgCl}_{2}, 1.2 \mathrm{~mm} \mathrm{NaH} \mathrm{PO}_{4}, 10 \mathrm{~mm}$ glucose, and $25 \mathrm{~mm} \mathrm{NaHCO}_{3}$ (pH 7.3-7.4, 290-300 mOsm).

Glycinergic evoked IPSCs (eIPSCs) were obtained from the motor neurons of the hypoglossal nucleus, as previously described (Petukhova et al., 2018). The DS3 Constant Current Isolated Stimulator (Digitimer) and a bipolar stimulating electrode were used for the induction of reliable elPSCs. CNQX (10 $\mu \mathrm{m})$, APV $(40 \mu \mathrm{m})$, and bicuculline (20 $\mu \mathrm{M})$ were routinely added to aCSF to block the glutamatergic and GABAergic synaptic transmission. Recording was conducted at room temperature in the whole-cell configuration with the holding potential of $0 \mathrm{mV}$ using the EPC-10 patchclamp amplifier (HEKA Elektronik). Patch electrodes were filled by the intracellular solution containing the following: $130 \mathrm{~mm}$ Cs-gluconate, $4 \mathrm{~mm}$ MgATP, $10 \mathrm{~mm}$ phosphocreatine, $0.3 \mathrm{~mm}$ GTP, $10 \mathrm{~mm}$ HEPES, $5 \mathrm{~mm}$ EGTA, and $4 \mathrm{~mm}$ $\mathrm{MgCl}_{2}$ (pH 7.3; 290 mOsm). Effects of Azo-NZ1 on the amplitudes of glycinergic elPSCs were examined at visible light and under illumination of aCSF by diode emitting $365 \mathrm{~nm}$ UV (Thorlabs).

\section{Data analysis and statistics}

To analyze the results of patch-clamp recordings, PatchMaster (HEKA Electronic), Origin 7.5 (OriginLabs), Excel 2016 (Microsoft), and Igor Pro 6.02 (WaveMetrics) software were employed to accomplish a statistical analysis of the data and to plot the graphs. Concentration-response curves using different concentrations of glycine were fitted using a nonlinear fitting routine of the Origin 7.5 software with the Hill equation:

$$
\mathrm{I}=\mathrm{I}_{\max } /\left(1+\left(\mathrm{EC}_{50} /[\mathrm{A}]\right)^{\mathrm{nH}}\right)
$$

where $I$ is the normalized current amplitude induced by the agonist at concentration $[A], I_{\max }$ is a maximal current induced at given cell, $n_{H}$ is the Hill coefficient and $\mathrm{EC}_{50}$ is the concentrations at which a half-maximum response was induced.

To quantify the inhibitory effect of Azo-NZ1, the following equation was used:

$$
\mathrm{I}_{\text {relative }}=\mathrm{I}_{2} / \mathrm{I}_{1} * 100 \% \text {, }
$$

where $I_{1}$ is the amplitude of the current before application of Azo-NZ1 (control) and $I_{2}$ is the amplitude of the current during the application of Azo-NZ1.
To quantify the effect of UV illumination, we used the following equation:

$$
\mathrm{I}_{\text {relative }}=\mathrm{I}_{3} / \mathrm{I}_{1} * 100 \% \text {, }
$$

where $I_{1}$ is the amplitude of the current before application of Azo-NZ1 (control), $I_{3}$ is the amplitude of the current during the application of Azo-NZ1 on UV illumination.

Taking into the account that, in the case of $\alpha 2 \mathrm{Z}, \alpha 2 \mathrm{M} /$ $\beta \mathrm{M}$, and $\alpha 1 \mathrm{H} \mathrm{G} 254 \mathrm{~A}$ receptors, desensitization was present, we suggested to estimate the UV effect with respect to the washout current. However, the hump current that appeared during the washing and the potentiation effect recorded on UV illumination (see also single-channel experiments) made the correct estimation of UV effect impossible. Because of these complications and the visibly prominent UV effect, we have arbitrarily fixed it at the level of the control current.

Concentration dependences of responses to different doses of Azo-NZ1 were fitted using a nonlinear fitting routine of the Origin 7.5 software (OriginLabs) with the Hill equation:

$$
\mathrm{I}=\mathrm{I}_{\max } /\left(1+\left([\mathrm{inh}] / \mathrm{IC}_{50}\right)^{\mathrm{nH}}\right) \text {. }
$$

The effect of Azo-NZ1 at each concentration was estimated through normalization of the current amplitude with respect to the amplitude of the control current. The point corresponding to the $1000 \mu \mathrm{m}$ was taken arbitrarily.

Data are represented as mean \pm SEM. Significance of differences was assessed using paired and unpaired $t$ tests, paired sample Wilcoxon signed-rank test. Differences were considered significant at $p<0.05$.

\section{Single-channel analysis}

Data were sampled at $10 \mathrm{kHz}$ using PatchMaster (HEKA Electronic) software. Further analysis was performed with the Nest-o-Patch program.

The amplitude histograms of simple openings were fitted with the sum of Gaussian functions (for $\alpha 2 \mathrm{M}$ GlyRs) or built based on the amplitudes of only completely resolved events ( $\alpha 1$ and $\alpha 1 \mathrm{H}$ G254A mutant GlyRs). Kinetic parameters have to be considered as "apparent" because of the effects of undetected shutting and opening (Colquhoun and Sigworth, 1995). The open probability ( $\mathrm{NP}_{\mathrm{o}}$ ) was calculated as the total open time of the channel during a recording divided by total time of the recording (Fucile et al., 1999).

\section{Drugs}

Stock solution of Azo-NZ1 (10 mm) was prepared using dimethylsulfoxide (DMSO) and then diluted to the final concentration by extracellular solution. All other drugs were obtained from Hello Bio and Tocris or Sigma-Aldrich. Stock solutions were prepared using DMSO or MilliQ water and kept at $-20^{\circ} \mathrm{C}$.

\section{Modelling}

GlyR structures

Four different GlyRs were considered (homomeric $\alpha 1 Z$,

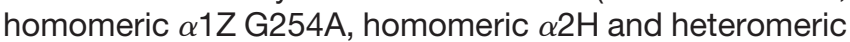


$\alpha 2 \mathrm{M} / \beta \mathrm{M})$. Structures of these GlyRs were modelled in the open channel state. For homomeric $\alpha 1 Z$ GlyR, we used the cryo-EM structure with glycine bound (PDB code 3JAE) from reference (Du et al., 2015). For homomeric $\alpha 1 Z$ GlyR G254A, we used the previous structure and introduced the G254A mutation in silico using the molefacture tool (version 1.3) in VMD (version 1.9.2; Humphrey et al., 1996). In doing so, we assume that the G254A $\alpha 1 Z$ structure is equivalent to the $\alpha 2 Z$ structure at the level of the pore-lining M2 helices.

Homology models were generated for homomeric $\alpha 2 \mathrm{H}$ and heteromeric $\alpha 2 \mathrm{M} / \beta \mathrm{M}$ GlyRs using SWISS-MODEL (Waterhouse et al., 2018). For the latter, being aware that the stoichiometry of heteromeric GlyRs is controversial, we decided to follow a recent study (Low et al., 2018) that proposes a $3 \alpha: 2 \beta$ stoichiometry with a $\beta-\alpha-\alpha-\beta-\alpha$ clockwise order. The overall sequence identity between the zebrafish $\alpha 1$ GlyR template and the target human $\alpha 2$ and mouse $\alpha 2$ and $\beta$ GlyRs is $89 \%, 87 \%$, and $55 \%$, respectively, whereas the sequence identity when only the pore-lining residues are considered is $95 \%, 95 \%$, and $38 \%$, respectively.

\section{Azo-NZ1 ligand}

The initial structures of the Azo-NZ1 compound (cisand trans- isomers) were created employing Avogadro (version 1.1.1; Hanwell et al., 2012). For each isomer, two 1,4-diazepine ring conformations, $\mathrm{M}$ and $\mathrm{P}$, were considered. These conformations differ in orientation (below or above the plane, respectively) of C3 and the phenyl substituent of C5. For classical benzodiazepines that bind to the canonical benzodiazepine allosteric site, the $\mathrm{M}$ conformation is the bioactive one (i.e., the one that shows higher affinity for receptor; Richter et al., 2012). However, it is not known a priori whether Azo-NZ1 would exhibit similar conformational preferences, since GlyR does not contain a benzodiazepine binding site. Therefore, all four possible ligand structures (cis/M, cis/ $\mathrm{P}$, trans/M and trans/P) were considered for docking. Optimized ligand structures were taken from (Maleeva et al., 2019).

\section{Docking calculations}

AutoDock Vina (version 1.1.2; Trott and Olson, 2010) was employed for ligand-receptor docking. Given that experimental data indicate that Azo-NZ1 binds inside the pore, we centered our search space around the M2 helices of the pore. A flexible docking approach was used, in which both the ligand and the pore-lining residues in positions 2', 6', 9', 13', and 16' were allowed to move. The maximum energy difference between the best and worst binding modes and the exhaustiveness were set to default values ( 3 and $8 \mathrm{kcal} / \mathrm{mol}$, respectively), while the maximum number of modes was increased to 20 to increase the docking sampling. This protocol was repeated 10 times starting with different random seeds, so that a total number of 200 poses was obtained for each of the four possible conformers of Azo-NZ1. The same protocol was previously used to study the binding determinants of AzoNZ1 in the pore of GABA $A_{A}$ and GABA $R$ (Maleeva et al., 2019).

We would like to note here that, on UV light irradiation, cis-Azo-NZ1 may either remain in the channel (adopting another binding conformation that unblocks the pore) or exit the pore (reaching the bath solution). The outcome will depend on the mean opening time of the channel and the dissociation rate of cis-Azo-NZ1. With the data at hand, we cannot distinguish between the two aforementioned possibilities. Therefore, we decided to perform docking calculations not only with trans-Azo-NZ1, but also with the cis isomer, to model the system immediately after UV irradiation. These calculations are aimed at investigating the change in the receptor-ligand binding mode right after trans-cis isomerization (both in terms of ligand position along the pore and interactions with the pore-lining residues).

In order to explore the possibility of Azo-NZ1 binding to other regions of the GlyR channel, we also performed "blind" docking calculations, in which the search space includes the whole receptor. The same docking protocol as for the calculations focused on the M2 helices of the pore was used. This strategy was recently used to uncover the putative binding site of another photochromic ligand, Glyght, to GlyRs (Gomila et al., 2020).

\section{Analysis of the docking results}

The docking poses of each conformer were analyzed separately. For simplicity, only the trans/P and cis/P results are discussed in the main text. The results of the corresponding $\mathrm{M}$ conformers are almost identical (data not shown) and, in the case of the cis isomer, the contribution of the $\mathrm{M}$ conformer is expected to be minor, as it is $3.5 \mathrm{kcal} / \mathrm{mol}$ less stable than cis/P. We used two different approaches to pinpoint the exact binding site of Azo-NZ1 in the M2 ion channel pore. First, we analyzed the number density of the sulfonate group of the ligand poses. Previous studies have successfully used this type of analysis to identify ligand binding sites in other ion channels (Raju et al., 2013; Maleeva et al., 2019). The underlying assumption is that regions of continuous density (or high occupancy) should represent regions of tighter binding. The number density value was computed using the Volmap plugin (Cohen et al., 2006) of VMD (Humphrey et al., 1996), as done previously (Maleeva et al., 2019). The second approach was based on the analysis of the interactions between the ligand and the receptor. Ligand binding poses were clustered using the quality threshold algorithm implemented in VMD (https:// github.com/luisico/clustering) and then the representative structure of the most populated cluster(s) was analyzed using the Binana algorithm (Durrant and McCammon, 2011). In the case of the blind docking calculations, we only analyzed the sulfonate number density, as these calculations are only intended to explore the possibility of alternative binding sites responsible for secondary effects of cis-AzoNZ1 on $\alpha 1$ GlyR, other than the main channel blocking activity. The $\mathrm{M}$ and $\mathrm{P}$ conformers of each isomer (cis/trans) were pooled together to carry out this analysis, to have more sampling. All the images of the modeling section were generated with either the UCSF Chimera package (Pettersen et al., 2004) or the VMD program (Humphrey et al., 1996).

\section{Results}

\section{Effect of Azo-NZ1 on $\alpha 1$ GlyR}

Azo-NZ1 is the azobenzene derivative of nitrazepam, capable to block $\mathrm{GABA}_{\mathrm{A}}$ receptor channels (Maleeva et 


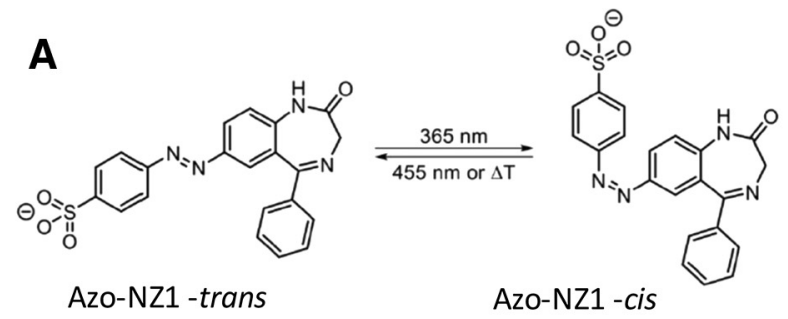

B

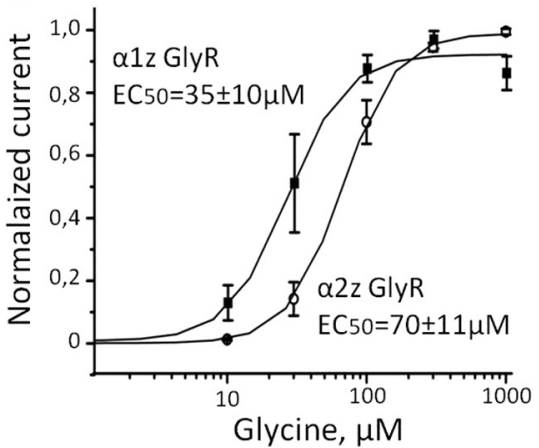

C

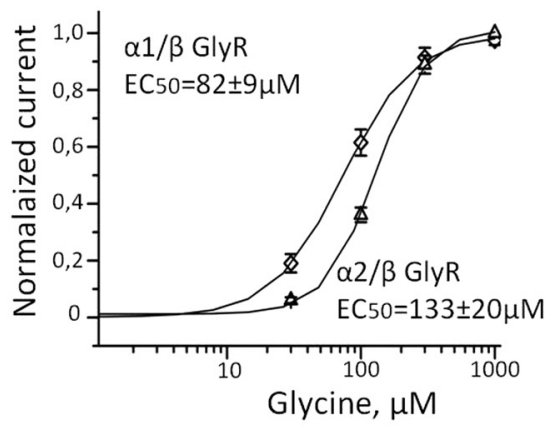

Figure 1. Schematic representation of Azo-NZ1 and cumulative curves of glycine-induced dose-response dependencies for different subtypes of GlyRs. A, Azo-NZ1 in its trans- and cis-configurations. B, Cumulative dose/response curves for homomeric $\alpha 1 Z$ (filled squares, $n=6$ ) and $\alpha 2 \mathrm{Z}$ GlyRs (empty circles, $n=6$ ). $\boldsymbol{C}$, Cumulative dose/response curves for heteromeric $\alpha 1 \mathrm{H} / \beta \mathrm{M}($ diamonds, $n=7$ ) and $\alpha 2 \mathrm{M} / \beta \mathrm{M}$ (triangles, $n=7$ ).

al., 2019). Ligand pharmacological activity is regulated with the azobenzene photochromic group, which changes its configuration to the cis isomer when illuminated with UV-light of $365 \mathrm{~nm}$. The back isomerization to the trans isomer can be triggered by blue or visible light (Fig. 1A).

To examine the effects of Azo-NZ1 on GlyRs, we first tested it at homomeric $\alpha 1$ zebrafish $(\alpha 1 Z)$ GlyRs expressed in $\mathrm{CHO}$ cells. The $\mathrm{EC}_{50}$ for glycine was $35 \pm 10$ $\mu \mathrm{M}(n=6$; Fig. $1 B)$, which is similar to the previous values obtained on BOSC 23 cells transiently expressing $\alpha 1 Z$ GlyRs (David-Watine et al., 1999; Fucile et al., 1999).

Co-application of Azo-NZ1 (50 $\mu \mathrm{m})$ with subsaturating concentration of glycine $(20 \mu \mathrm{M})$ resulted in a decrease of current amplitude by $34 \pm 2 \%$ of control (Fig. $2 A$ ), with an $\mathrm{IC}_{50}$ for Azo-NZ1 of $78 \pm 8 \mu \mathrm{M}$ and $\mathrm{n}_{\mathrm{H}}=1.4 \pm 0.25(n=6$; Fig. 2C). Illumination with UV light produced a slightly additional inhibition (Fig. 2A). Importantly, the inhibitory action of Azo-NZ1 decreased at elevation of the agonist concentration and the photochromic compound did not produce any detectable effect at $\alpha 1 Z$ GlyRs activated by saturating concentration of glycine (300 $\mu \mathrm{m}$; Fig. 2B). Similar results were obtained on homomeric human GlyR receptors formed by $\alpha 1$ subunits, using $30 \mu \mathrm{m}$ glycine and $50 \mu \mathrm{m}$ Azo-NZ1 (Fig. 2E).

In rodents, expression of different GlyR subtypes is developmentally regulated: $\alpha 2$ GlyRs are predominant at birth, but during the two weeks of postnatal life $\alpha 1$ expression increases dramatically and becomes predominant in adult stages of life (Akagi and Miledi, 1988; Becker et al., 1988; Malosio et al., 1991). In addition, the postsynaptic clustering of GlyRs requires the $\beta$ subunit (Meyer et al., 1995), and in the CNS of adult vertebrates, glycinergic synaptic transmission is predominantly provided by heteromeric $\alpha 1 / \beta$ receptors (Becker et al., 1988). For some compounds, the pharmacological profile of heteromeric receptors may be different from the homomeric ones (Pribilla et al., 1992). Thus, we performed analysis aimed to determine whether incorporation of $\beta$ subunits will change the profile of $\alpha 1$ GlyRs interaction with AzoNZ1.

Addition of Azo-NZ1 at $50 \mu \mathrm{m}$ concentration caused inhibition of currents mediated by heteromeric $\alpha 1 \mathrm{H} / \beta \mathrm{M}$ GlyRs to $73 \pm 3 \%$ with a minor strengthening of inhibition at UV illumination to $64 \pm 4 \%(n=7$; Fig. $2 D, E)$. In the same set of experiments, monomeric $\alpha 1 \mathrm{H}$ GlyRs were inhibited by Azo-NZ1 to $78 \pm 4 \%$, with minor UV effect, to $64 \pm 3 \%$ ( $n=3$; Fig. $2 E)$.

These observations demonstrate that Azo-NZ1 weakly inhibits homomeric $\alpha 1 \mathrm{Z}, \alpha 1 \mathrm{H}$ and heteromeric $\alpha 1 \mathrm{H} / \beta$ GlyRs activated by low concentrations of glycine and does not block $\alpha 1$ GlyRs in the presence of high concentrations of the agonist.

\section{Effect of Azo-NZ1 on $\alpha 2$ GlyR}

Analysis of concentration dependencies revealed that $\mathrm{EC}_{50}$ for glycine of $\alpha 2 Z$ GlyRs is $70 \pm 11 \mu \mathrm{M}(n=6$; Fig. $1 B$ ). As this value is higher than for $\alpha 1$ subunits, the analysis of Azo-NZ1 action on $\alpha 2$ GlyR was performed at glycine concentrations of 50 and $300 \mu \mathrm{m}$ (non-saturating and saturating, respectively).

The effect of Azo-NZ1 at $\alpha 2 Z$ GlyRs was drastically different from the one observed at $\alpha 1 \mathrm{Z}$ and $\alpha 1 \mathrm{H}$ GlyRs. The current amplitude was strongly inhibited by application of Azo-NZ1 $(50 \mu \mathrm{M})$ in trans-conformation, to $9 \pm 5 \%(n=3$; Fig. $3 A, C)$, while the effect was virtually abolished on 
A alpha1Z GlyRs

Glycine $20 \mu \mathrm{M}$

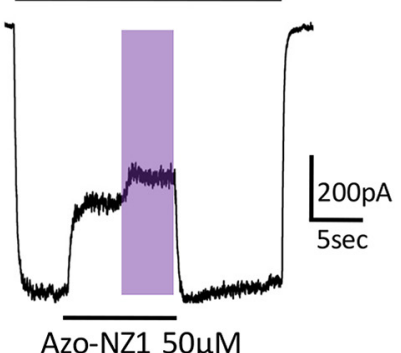

Azo-NZ1 50 $\mu \mathrm{M}$

D alpha1/beta GlyRs

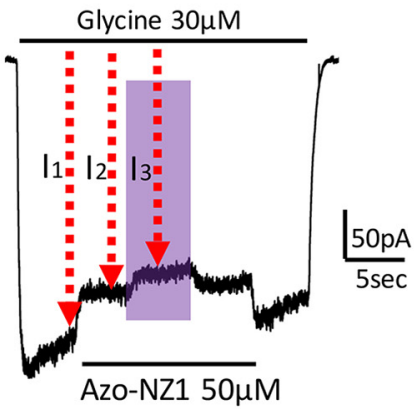

B

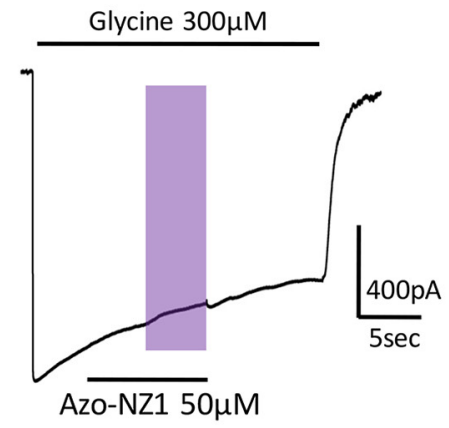

E
C

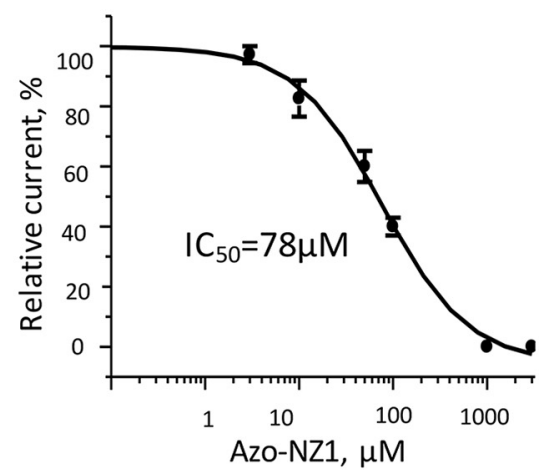

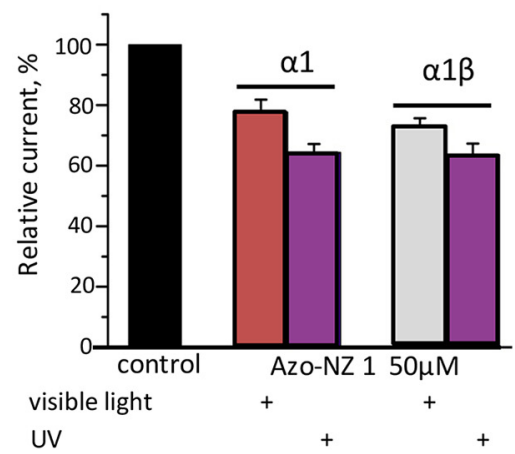

Figure 2. Interaction of Azo-NZ1 with homomeric $\alpha 1$ and heteromeric $\alpha 1 / \beta$ GlyRs. $\boldsymbol{A}$, Representative trace of the $\alpha 1 Z$ GlyRs-mediated current induced by application of glycine $20 \mu \mathrm{m}$ (below $\mathrm{EC}_{50}$ ) and by mixture of glycine $20 \mu \mathrm{m}$ with Azo-NZ1 $50 \mu \mathrm{M}$ at visible light and on illumination with UV light (here and in other figures, UV illumination is shown by violet rectangles). $\boldsymbol{B}$, Representative trace of $\alpha 1 Z$ current induced by saturating glycine concentration, $300 \mu \mathrm{m}$, and by a mixture of glycine $300 \mu \mathrm{m}$ with Azo-NZ150 $\mu \mathrm{m}$ at visible light and on illumination with UV light. Note the absence of Azo-NZ1 inhibitory effect. $\boldsymbol{C}$, Cumulative dose-response curve for Azo-NZ1 action on $\alpha 1 Z$ GlyRs at glycine concentration of $20 \mu \mathrm{M}$. IC $\mathrm{I}_{50}=78 \pm 8 \mu \mathrm{M}, \mathrm{n}_{\mathrm{H}}=1.4 \pm 0.25(n=6) ; \mathrm{V}_{\text {hold }}=-30 \mathrm{mV}$. $\boldsymbol{D}$, Representative trace of $\alpha 1 / \beta$-mediated current induced by application of glycine $30 \mu \mathrm{m}$ and by a mixture of glycine with Azo-NZ1 $(50 \mu \mathrm{M}) . I_{1}$ and $I_{2}$ are the amplitudes of currents used to calculate the Azo-NZ1 inhibitory effect, $I_{1}$ and $I_{3}$ were used to calculate the UV effect. $E$, Relative amplitudes of $\alpha 1 \mathrm{H}$ and $\alpha 1 \mathrm{H} / \beta \mathrm{M}$ currents induced by application of glycine $30 \mu \mathrm{M}$ or by mixture of glycine with Azo-NZ1 $50 \mu \mathrm{m}$. Data from three to seven cells.

illumination with UV light. Contrary to $\alpha 1$ GlyRs, transAzo-NZ1 still strongly inhibited ionic currents when it was co-applied with saturating concentration of glycine. Under visible light current amplitude decreased to $24 \pm 9 \%(n=3$; Fig. $3 B, C)$.

To further clarify the properties of the Azo-NZ1, we conducted an analysis of the concentration and voltage dependencies of its inhibitory action on the $\alpha 2$ GlyRs. Analysis of concentration dependencies revealed that $\mathrm{IC}_{50}$ for Azo-NZ1 at $\alpha 2 Z$ GlyRs was $9 \pm 2$ $\mu \mathrm{M}$, which was considerably lower than for $\alpha 1 Z$ GlyRs $(n=4$; Fig. $3 D)$. Voltage dependence of transAzo-NZ1 interaction with $\alpha 2 Z$ GlyRs was thus performed using $20 \mu \mathrm{m}$ the photochrome and $50 \mu \mathrm{m}$ glycine. At $30 \mathrm{mV}, \alpha 2 Z$-mediated currents were inhibited to $49 \pm 2 \%$, while at $+30 \mathrm{mV}$ to $29 \pm 3 \%(n=8, p \leq 0.001$; Fig. 3E).

Voltage dependence and strong inhibitory potency even at saturating concentrations of the agonist strongly suggest that trans-Azo-NZ1 interacts with the ion channel pore of the receptors formed by $\alpha 2$ subunits.
Next, we analyzed the effect of Azo-NZ1 on heteromeric $\alpha 2 \mathrm{M} / \beta \mathrm{M}$ GlyRs. Analysis of concentration-dependencies revealed that the incorporation of $\beta$ subunit lowers the sensitivity of $\alpha 2$ receptors to glycine. The $\mathrm{EC}_{50}$ for heteromeric $\alpha 2 \mathrm{M} / \beta \mathrm{M}$ GlyRs was $133 \pm 20 \mu \mathrm{M}(n=7$; Fig. $1 C)$. Hence, analysis of the action of Azo-NZ1 on this heteromeric GlyR was conducted using $100 \mu \mathrm{m}$ glycine as "nonsaturating" concentration.

In the presence of $50 \mu \mathrm{m}$ Azo-NZ1, glycine-induced currents mediated by $\alpha 2 \mathrm{M} / \beta \mathrm{M}$ GlyRs were inhibited to $48 \pm 6 \%(n=8)$, while currents mediated by homomeric $\alpha 2 \mathrm{M}$ GlyRs were inhibited to $23 \pm 3 \%(n=10$; Fig. $3 F, G)$. These data suggest that incorporation of the $\beta$ subunit causes a decrease in the inhibitory action of Azo-NZ1. Illumination with UV light, converting Azo-NZ1 to cisform, caused recovery of the current amplitudes to nearly control values. Interestingly, in some experiments, during the first moments of the UV illumination, the current increased to values higher than predicted, showing a transient "potentiation," which could be masked by desensitization. 


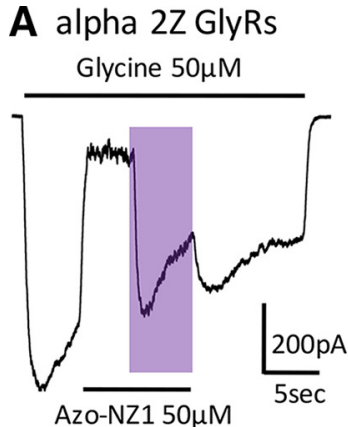

D

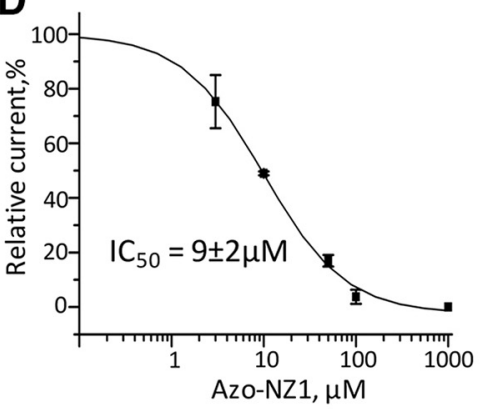

F alpha2/beta GlyRs

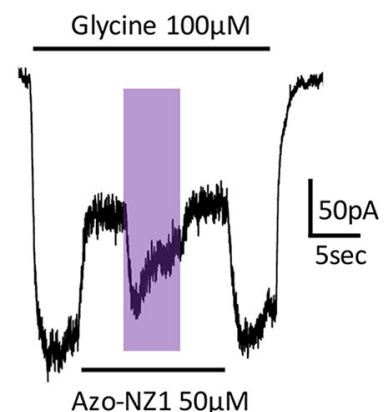

B

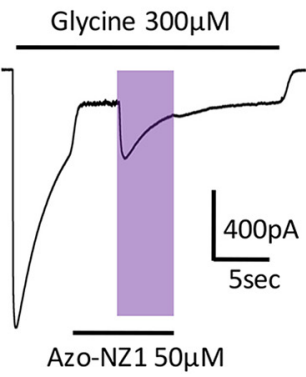

E

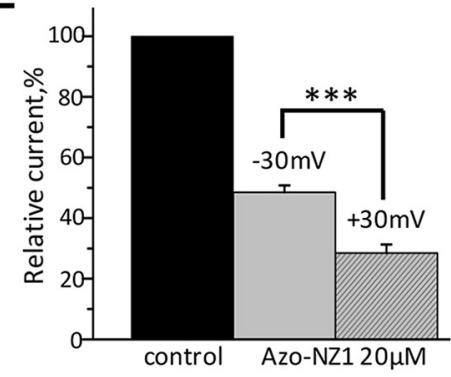

G

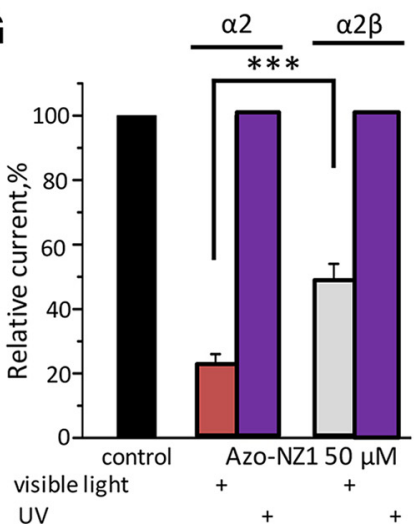

Figure 3. Azo-NZ1 is a potent UV-controllable inhibitor of $\alpha 2$ GlyRs. $\boldsymbol{A}$, Representative trace of $\alpha 2 Z$ GlyRs current induced by application of glycine $50 \mu \mathrm{m}$ or by a mixture of glycine with Azo-NZ1 $50 \mu \mathrm{m}$ at visible light or at UV illumination. $\boldsymbol{B}$, Representative trace of $\alpha 2 Z$ current induced by application of glycine $300 \mu \mathrm{m}$ or by a mixture of glycine with Azo-NZ1 $50 \mu \mathrm{m}$ at visible light or on UV illumination. C . Cumulative data on relative amplitude of Azo-NZ1 inhibition (50 $\mu \mathrm{M})$ of glycine-induced currents (50 and $300 \mu \mathrm{M})$ at visible light and on UV illumination. $\boldsymbol{D}$, Cumulative dose-response curve for Azo-NZ1; sigmoidal fitting yielded $\mathrm{IC}_{50}=9 \pm 2 \mu \mathrm{M} ; \mathrm{n}_{\mathrm{H}}=$ $1.02 \pm 0.3(n=4) ; V_{\text {hold }}=-30 \mathrm{mV}$. $\boldsymbol{E}$, Cumulative data on the voltage dependence of Azo-NZ1 interaction with $\alpha 2 Z$ GlyRs (black column, relative amplitude of the current in control; gray column, on application of Azo-NZ1 $20 \mu \mathrm{m}$ at $\mathrm{V}_{\text {hold }}=-30 \mathrm{mV}$; dashed column, on application of Azo-NZ1 $20 \mu \mathrm{M}$ at $\mathrm{V}_{\text {hold }}=+30 \mathrm{mV}$ ); $n=6-8$ cells, ${ }^{\star * *} p \leq 0.001$. $\boldsymbol{F}$, Representative trace of $\alpha 2 \mathrm{M} / \beta \mathrm{M}$-mediated current induced by glycine $100 \mu \mathrm{m}$ or by mixture of glycine with Azo-NZ1 $(50 \mu \mathrm{M})$ at visible light or on UV illumination. G, Relative amplitudes of current mediated by $\alpha 2 \mathrm{M}$ and $\alpha 2 \mathrm{M} / \beta \mathrm{M}$ receptors during the application of Azo-NZ1 $50 \mu \mathrm{M}$ at visible light and on illumination with UV light $\left(n=8-10\right.$ cells, $\left.{ }^{* *} p \leq 0.001\right)$.

\section{Single-channels analysis of Azo-NZ1 interaction with GlyRs}

In order to resolve in more detail the action of Azo-NZ1 on GlyR, we performed single-channel recordings of $\alpha 1 \mathrm{H}$ and $\alpha 2 \mathrm{M}$ GlyRs from membrane patches in outside-out configuration on application of $5 \mu \mathrm{m}$ glycine (Fig. 4). In the control conditions, when only glycine was applied, UV illumination did not change the activity of GlyR channels (Fig. 4E).

In outside patches expressing $\alpha 1 \mathrm{H}$ GlyRs, application of Azo-NZ1 (50 $\mu \mathrm{M})$ in trans-state provoked a slight decrease of the open probability $\left(\mathrm{NP}_{\mathrm{o}}\right.$ ) of the channels (from
$0.013 \pm 0.002$ to $0.011 \pm 0.002$ ), which was strengthened by UV illumination (to $0.007 \pm 0.002$; Fig. $4 A-D$ ). On outside-out patches expressing $\alpha 2 \mathrm{M}$ GlyRs, Azo-NZ1 (20 and $50 \mu \mathrm{m})$ in trans-state induced a prominent decrease of the open probability of channels, which was accompanied by an increase in the flickering of channels openings. UV illumination caused restoration of $\mathrm{NP}_{\mathrm{o}}$ and even its transient increase, particularly, in the case of application of Azo-NZ1 at $50 \mu \mathrm{m}$ concentration. We have estimated $\mathrm{NP}_{\text {o }}$ of $\alpha 2$ GlyR channels in all conditions: in control, it was $0.64 \pm 0.09$; on co-application of glycine with $20 \mu \mathrm{M}$ 
alpha1 GlyRs

A

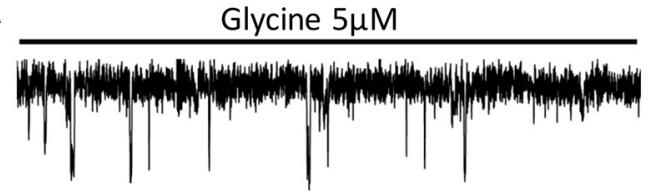

B

Glycine $5 \mu \mathrm{M}+$ Azo-NZ1 50 $\mu \mathrm{M}$

C

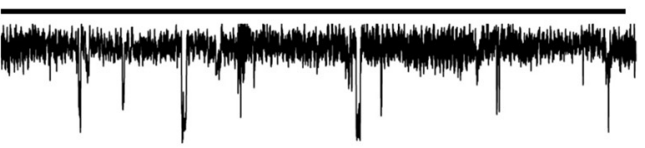

Glycine $5 \mu \mathrm{M}+$ Azo-NZ1 50 $\mu \mathrm{M}$

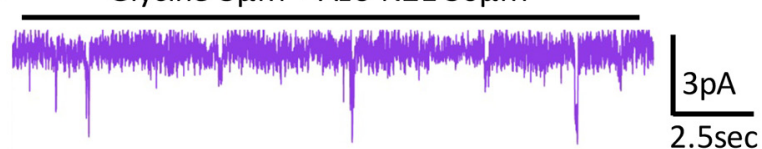

D

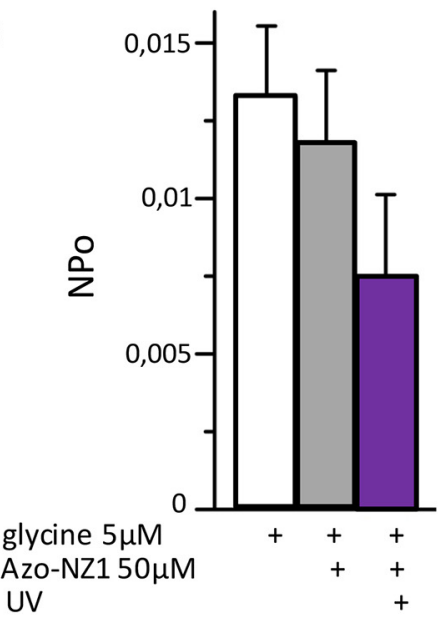

alpha2 GlyRs

E

Glycine $5 \mu \mathrm{M}$

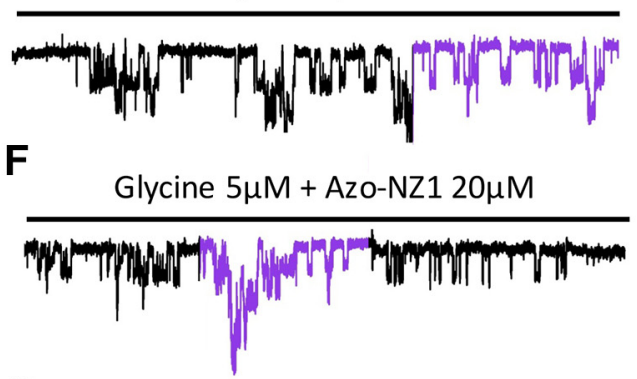

G
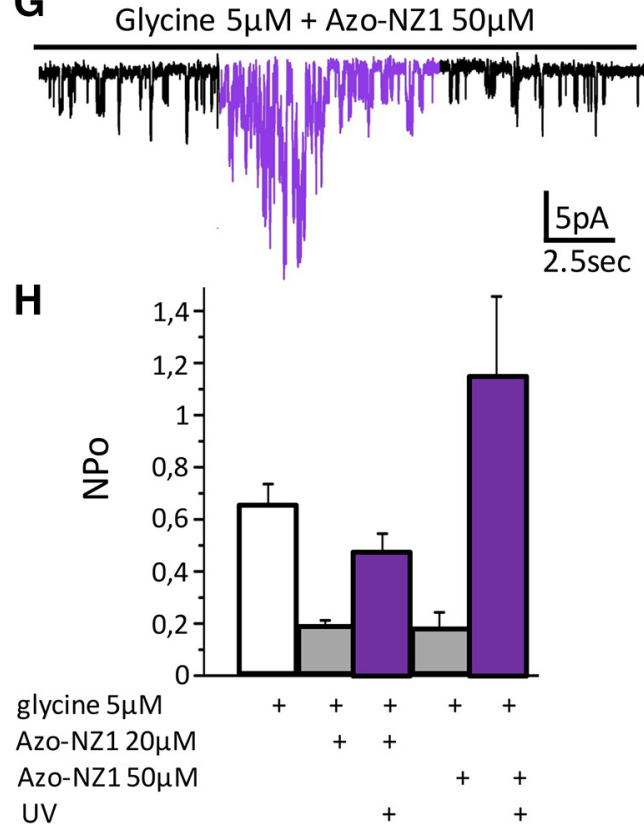

Figure 4. Single-channels recording of $\alpha 1 \mathrm{H}$ and $\alpha 2 \mathrm{M}$ GlyRs interaction with Azo-NZ1 in outside-out configuration of patch-clamp technique. $\boldsymbol{A}$, Representative recording of single-channel activity of $\alpha 1 Z$ GlyRs in control, currents were induced by application of $5 \mu \mathrm{m}$ glycine, $V_{\text {hold }}=-30 \mathrm{mV}$. B. Channel activity in the solution containing a mixture of glycine $5 \mu \mathrm{m}$ with trans-Azo-NZ1 $50 \mu \mathrm{m}$ at visible light and (C) on UV illumination (cis-Azo-NZ1). $\boldsymbol{D}, \mathrm{NP}_{\mathrm{o}}$ for $\alpha 1 \mathrm{H}$ GlyRs in control conditions (white column), when glycine $5 \mu \mathrm{M}$ was co-applied with Azo-NZ1 $50 \mu \mathrm{M}$ under visible light (gray column), or on UV illumination (violet column). $\boldsymbol{E}$, Channel activation was induced by application of $5 \mu \mathrm{M}$ glycine, $V_{\text {hold }}=-30 \mathrm{mV}$. F, A mixture of glycine $5 \mu \mathrm{m}$ with Azo-NZ1 $20 \mu \mathrm{m}$ was applied, $\mathrm{V}_{\text {hold }}=-30 \mathrm{mV}$; the part of the trace recorded on illumination with UV light is colored in violet. $G$, A mixture of glycine $5 \mu \mathrm{m}$ with Azo-NZ1 $50 \mu \mathrm{m}$ was applied, $\mathrm{V}_{\text {hold }}=-30 \mathrm{mV}$; the part of the trace recorded on UV illumination is colored in violet. $\boldsymbol{H}, \mathrm{NP}_{\mathrm{o}}$ for $\alpha 2 \mathrm{M}$ GlyRs in control conditions (white column), when glycine $5 \mu \mathrm{M}$ was co-applied with Azo-NZ1 $20 \mu \mathrm{m}$ or $50 \mu \mathrm{m}$ under visible light (gray columns), or on UV illumination (violet columns).

Azo-NZ1, it decreased to $0.19 \pm 0.02$; UV light restored $\mathrm{NP}_{\mathrm{o}}$ almost to the control level, to $0.47 \pm 0.07$. Application of Azo-NZ1 in higher concentration $(50 \mu \mathrm{M})$ decreased $\mathrm{NP}_{\circ}$ to $0.18 \pm 0.06$, while UV light increased it to $1.14 \pm 0.3$ (Fig. $4 E-H$ ).

These results confirm the observations obtained at whole-cell recordings that on $\alpha 2$ GlyRs transitions of AzoNZ1 from trans- to cis-configuration results in transient potentiation of glycine-induced currents. Molecular basis of this phenomenon needs further analysis.

\section{Effect of Azo-NZ1 on synaptic GlyRs in hypoglossal motoneurons of brainstem slices}

We next asked, whether Azo-NZ1 will modulate in a light-dependent manner the function of synaptic glycinergic currents. For this analysis, hypoglossal motoneurons in brainstem slices, possessing powerful glycinergic synaptic inputs (Mukhtarov et al., 2005) were selected. As experiments with heterologous expression of different GlyR subunits demonstrated that Azo-NZ1 strongly modulates function of heteromeric $\alpha 2 / \beta$ GlyR channels subunits, while being weakly efficient on the $\alpha 1 / \beta$ GlyRs, we performed analysis on brain slices from young mice (P4-P8), expressing at this age both $\alpha 1$ and $\alpha 2$ GlyR subunits (Malosio et al., 1991; Singer et al., 1998).

The effect of Azo-NZ1 on the amplitude of evoked glycinergic IPSCs (elPSC) was analyzed in the presence of glutamate receptor and $\mathrm{GABA}_{A}$ receptor antagonists. In control conditions, the amplitude of elPSCs varied from 40 to $800 \mathrm{pA}$ in the different cells. The effects of Azo-NZ1 on the glycinergic 
A

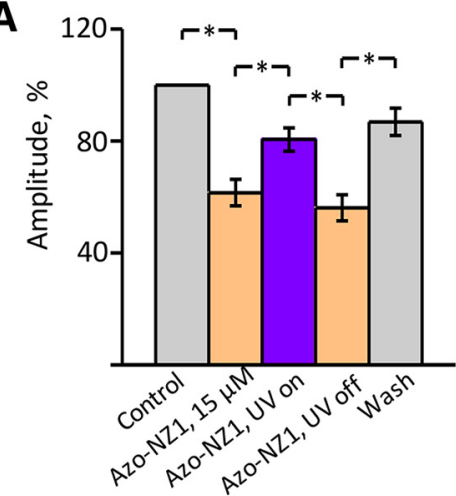

C

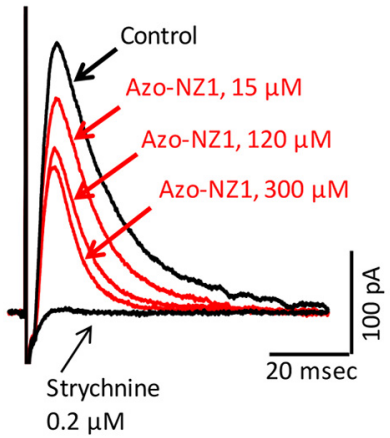

B

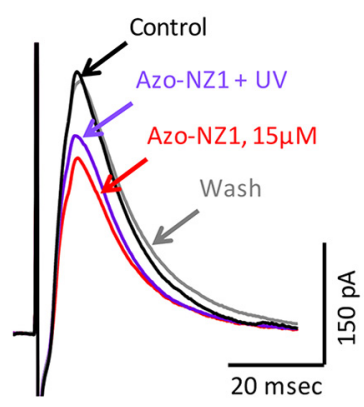

D

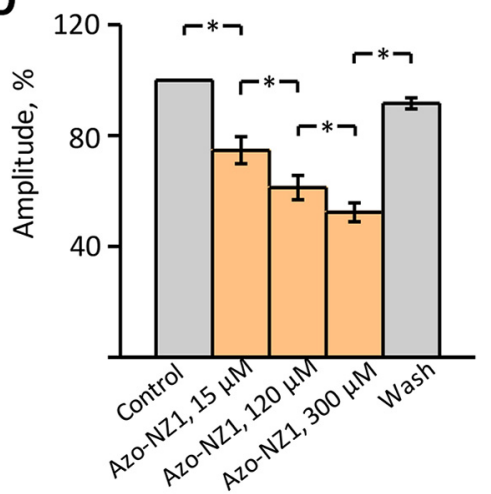

Figure 5. Azo-NZ1 is a photoswitchable modulator of the evoked glycinergic IPSCs (elPSCs) in the mouse hypoglossal motoneurons. $\boldsymbol{A}$, Relative elPSC amplitudes under the action of Azo-NZ1 (15 $\mu \mathrm{M})$ at visible light and on UV illumination, summary of data from eight neurons (mean percentage $\pm \mathrm{SEM}$ ). *, significant difference with $p<0.05$ (paired sample Wilcoxon signed-rank test). $\boldsymbol{B}$, Superimposed traces of elPSCs in control condition, after addition to aCSF of $15 \mu \mathrm{M}$ Azo-NZ1, during illumination of Azo-NZ1 containing aCSF by UV (365 nm) and after washing. Each trace represents the average of 18 individual elPSCs, obtained from a P8 mouse. $\boldsymbol{C}$, Superimposed traces illustrating an example of elPSC amplitudes decreasing under 15, 120, and $300 \mu \mathrm{M}$ Azo-NZ1 and complete suppression of events by $0.2 \mu \mathrm{M}$ strychnine. Each trace represents average of 12 individual eIPSCs, obtained from a P6 mouse. $\boldsymbol{D}$, Decreasing of glycinergic elPSC amplitude under the action of Azo-NZ1 at the concentrations of 15, 120, and $300 \mu \mathrm{M}$, summary of data from six neurons (mean percentage \pm SEM). *, significant difference with $p<0.05$ (paired sample Wilcoxon signed-rank test).

elPSCs in the mouse hypoglossal motoneurons are summarized in Figure 5. Addition of Azo-NZ1 at $15 \mu \mathrm{m}$ concentration caused a decrease of the mean amplitude of eIPSCs to $61.5 \pm 4.7 \%$ compared with control after illumination of aCSF with Azo-NZ1 by UV, relative amplitude increased to $80.5 \pm 4.2 \%$, while under visible light it decreased to $56.1 \pm 4.6 \%$ and during washing by control solution it recovered to $86.8 \pm 4.9 \%$ ( $n=8$; Fig. $5 A, B)$. Strychnine $(0.2 \mu \mathrm{M})$ completely abolished eIPSCs, confirming the glycinergic nature of the currents (Fig. 5C).

Analysis of the effect of different trans-Azo-NZ1 concentrations on glycinergic eIPSCs showed that the amplitude of currents decreased with elevation of the doses. However, the inhibition did not exceed $50 \%$ even at high concentrations of the compound (Fig. 5C,D). Thus, the mean relative amplitudes were $61.2 \pm 4.4 \%$ and $52.3 \pm 3.4 \%$ in the presence of, respectively, 120 and $300 \mu \mathrm{m}$ trans-Azo-NZ1 $(n=6)$.

\section{Mutagenesis analysis of the Azo-NZ1 interaction site at GlyRs}

Previously, Azo-NZ1 was shown to be a pore-blocker of GABARs (Maleeva et al., 2019). The high level of homology between $\alpha 2$ GlyRs channels and GABARs at the level of TM2 helix, as well as the single-channel recordings demonstrating increased flickering of $\alpha 2$ channels on application of trans-Azo-NZ1 (Fig. 4E-H), suggests that Azo-NZ1 interacts with the pore of $\alpha 2$ GlyRs and that this interaction is strongly reduced in $\alpha 1$ GlyRs.

It is well established that the amino acid sequences of ion pore-forming transmembrane domains (TMDs) of $\alpha 1$ and $\alpha 2$ subunits differ by one amino acid, which results in subunit-specific action of such inhibitory compounds as NFA and cyanotriphenylborate (CTB; Rundström et al., 1994; Maleeva et al., 2017). To clarify whether Azo-NZ1 interacts with the pore of $\alpha 2$ GlyRs, we generated mutant G254A $\alpha 1$ receptors that contained an amino acid substitution at position 2' in the pore-forming TM2 domain. Amino acid sequences of TM2 domains of $\alpha 1$ and $\alpha 2$ subunits differ only in this position, thus G254A substitution converts the pore of $\alpha 1$ receptor into the one of $\alpha 2$. As illustrated in Figure 6B,C, the profile of $\alpha 1 \mathrm{H}$ G254A GlyRs interaction with Azo-NZ1 was distinct from the one of $\alpha 1 \mathrm{H}$ and Z GlyRs (Fig. 2) and closely resembled that of $\alpha 2 \mathrm{M}$ and $Z$ receptors (Fig. 3). The amplitude of glycine-evoked 
A

$$
\begin{array}{llllll}
-1 & 2^{\prime} & 6^{\prime} & 9^{\prime} & 13^{\prime} & 16^{\prime}
\end{array}
$$

alpha1 PARVGLGITTVLTMTTQSSGS alpha2 PARVALGITTVLTMTTQSSGS beta

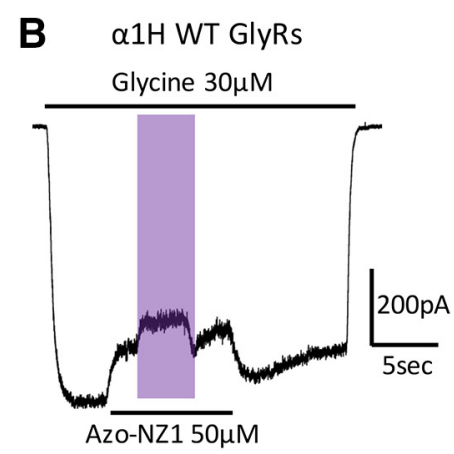

E

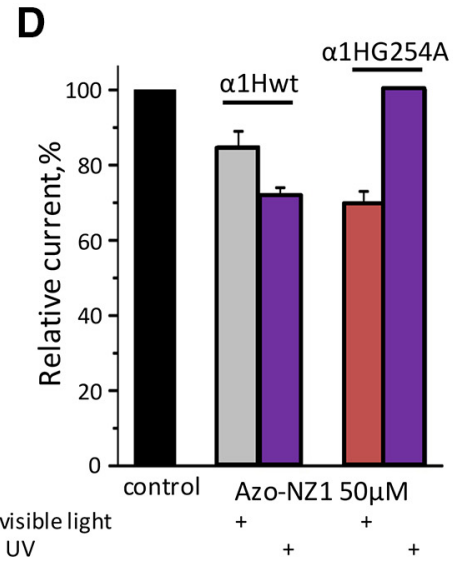

C a1H G254A GlyRs

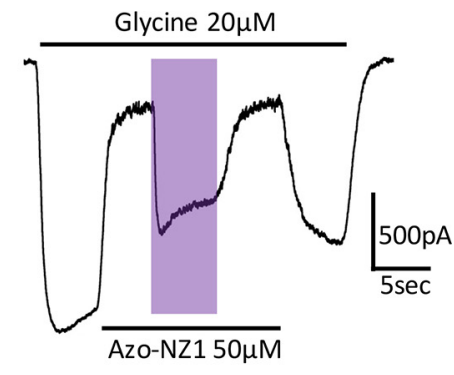

H

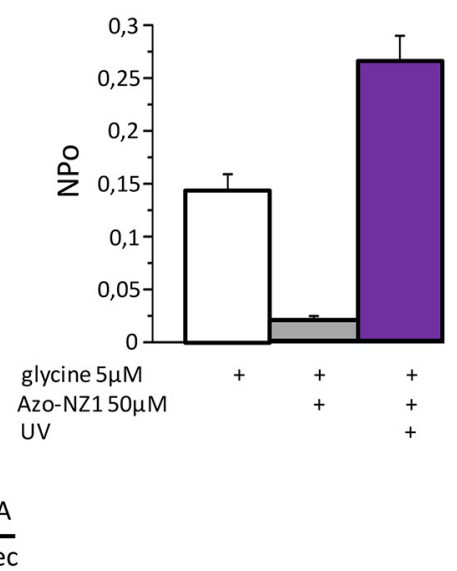

Figure 6. Comparison of the interaction of $\alpha 1$ WT GlyRs and $\alpha 1$ G254A GlyR with Azo-NZ1. A, Amino acid sequences of TM2 domains of $\alpha 1, \alpha 2$, and $\beta$ subunits of GlyR. $\boldsymbol{B}$, Representative trace of the $\alpha 1 \mathrm{H}$ GlyR current induced by application of glycine $30 \mu \mathrm{m}$ and by a mixture of glycine $30 \mu \mathrm{m}$ with Azo-NZ1 $50 \mu \mathrm{m}$ at visible light and on UV illumination. C, Representative trace of the $\alpha 1 \mathrm{H}$ G254A GlyRs current induced by application of glycine $20 \mu \mathrm{m}$ and by mixture of glycine with Azo-NZ1 $50 \mu \mathrm{m}$ at visible light and on UV illumination. $\boldsymbol{D}$, Relative amplitudes of $\alpha 1 \mathrm{H}$ and $\alpha 1 \mathrm{H}$ G254A GlyR currents in control (black column), and during application of Azo-NZ1 $50 \mu \mathrm{m}$ at visible light (gray and red columns, respectively) and at UV illumination (violet columns). E, Representative singlechannel recording of $\alpha 1 \mathrm{H}$ G254A currents in control; during application of Azo-NZ1 $50 \mu \mathrm{m}$ at visible light (F); on illumination with UV light $(\boldsymbol{G}) . \boldsymbol{H}, \mathrm{NP}_{\circ}$ for $\alpha 1 \mathrm{H}$ G254A GlyRs in control conditions (white column), when glycine $5 \mu \mathrm{m}$ was co-applied with Azo-NZ1 $50 \mu \mathrm{m}$ under visible light (gray column) or on UV illumination (violet column).

currents decreased on trans-Azo-NZ1 application to $70 \pm 3 \%(n=7)$. This effect was abolished by UV illumination (Fig. $6 B-D$ ), similarly to what was seen at $\alpha 2 \mathrm{M}$ GlyRs (Fig. 3).

These results were confirmed in a series of experiments on single-channel recordings from outside-out patches expressing $\alpha 1 \mathrm{H}$ G254A mutant GlyRs. Application of AzoNZ1 (50 $\mu \mathrm{M})$ decreased NPo of $\alpha 1 \mathrm{H}$ G254A channels from $0.14 \pm 0.019$ to $0.022 \pm 0.002$, while UV light induced elevation of channels activity, and $\mathrm{NP}_{\mathrm{o}}$ increased 10 -fold to $0.26 \pm 0.025$ (Fig. $6 E-H$ ). Thus, our single-channel experiments reproduced results obtained by whole-cell recordings, confirming a crucial role of the alanine ring at the 2' position of GlyR channel pore in the different interaction of Azo-NZ1 with $\alpha 1$ and $\alpha 2$ GlyRs and in the different UV effect registered for these receptors.

\section{Modelling of Azo-NZ1 molecular interactions with GlyR}

To understand the mechanism of action of Azo-NZ1 at atomic level, we performed molecular docking calculations for three different GlyRs: homomeric $\alpha 1 Z$ wild-type, $\alpha 1 Z$ G254A mutant (equivalent to $\alpha 2 Z$ in the pore) and heteromeric $\alpha 2 \mathrm{M} / \beta \mathrm{M}$ (see Materials and Methods). All receptors were modelled in the open channel state. In view of the importance of the 2' pore-lining amino acids indicated by the experimental data (Fig. 6), the docking simulation was conducted in the transmembrane region of the channel pore (Fig. 7A).

The docking results for trans-Azo-NZ1 show a similar binding pattern for all three GlyRs (Extended Data Fig. 71). Trans-Azo-NZ1 binds preferably between the 2'-16' pore region, with two possible orientations of the sulfonate group (Extended Data Figs. 7-2, 7-3): either with the sulfonate group between the 13'-16' rings (hereafter, up configuration) or with sulfonate in the 2 ' region (i.e., down configuration).

In contrast, the sulfonate density of cis-Azo-NZ1 was found to be more dispersed (Extended Data Fig. 7-4), indicating weaker binding. Moreover, it is mainly concentrated in the 6'-13' region (Extended Data Fig. 7-5), where the diameter of the pore is wider and thus more difficult to block completely. Analysis of the binding pose of the cis isomer (Fig. 7C; Extended Data Fig. 7-6) shows 


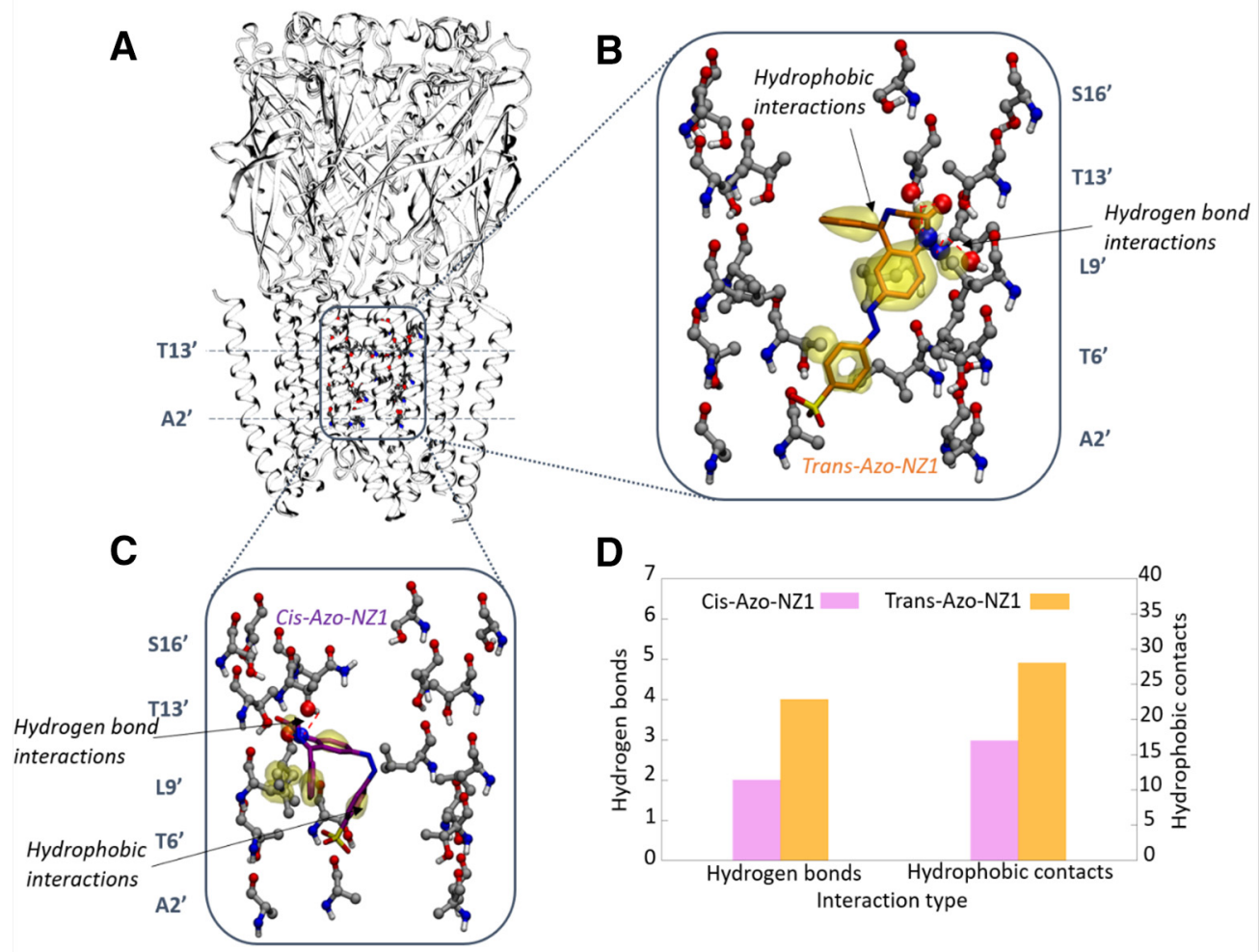

Figure 7. Results of the docking simulation of Azo-NZ1 in the transmembrane part domain of the $\alpha 1$ G254A GlyR mutant mimicking the $\alpha 2$ GlyR pore. Results for wild-type $\alpha 1$ GlyR and the heteromeric $\alpha 2 / \beta$ GlyR are shown in Extended Data Figures 7-1, 7-2, 7-3, 7-4, 7-5, 7-6. A, Tridimensional view of the $\alpha 1$ G254A mutant GlyR structure (open state). The A2' and T13' positions are marked with dashed lines. The sulfonate group of Azo-NZ1 can bind with two orientations: up (bound at 13') and down (bound at 2'); see Extended Data Figures 7-1, 7-3. B, Binding pose of the trans isomer in the transmembrane domain. The sulfonate group is in the down configuration; see Extended Data Figure 7-3 for the corresponding poses in wild-type $\alpha 1$ GlyR and the heteromeric $\alpha 2 / \beta$ GlyR. Hydrophobic interactions between Azo-NZ1 and the receptor residues are marked as yellow transparent surfaces and hydrogen bond interactions are represented with red dashed lines. $\boldsymbol{C}$, Binding pose of the cis isomer in the transmembrane domain. The sulfonate group is in the down configuration; see Extended Data Figure 7-6 for the corresponding poses in wild-type $\alpha 1$ GlyR and the heteromeric $\alpha 2 / \beta$ GlyR. The representations used for hydrophobic and hydrogen bond interactions are the same as for the trans isomer. $\boldsymbol{D}$, Relative number of intermolecular interactions formed by the cis and trans isomers of Azo-NZ1 with the pore-lining residues in $\alpha 1$ G254A mutant GlyR.

that, because of its staple-like shape, the ligand is slightly shifted upwards with respect to the trans isomer and thus it cannot block chloride conduction as effectively as the trans isomer (either sterically or electrostatically). Moreover, the number of interactions with the receptor is decreased for cis-Azo-NZ1 compared with the trans isomer (Fig. 7D), indicating that the ligand will have a higher probability of dissociation on UV irradiation. Therefore, our simulations predict that on isomerization, even if cis-Azo-NZ1 remains bound, it would be less capable to block the pore of the GlyRs compared with the trans isomer.

Out of the two aforementioned orientations of transAzo-NZ1 in the pore, the up pose is unlikely to block conduction. On one hand, the negatively charged sulfonate group is located in the wider 16' region and thus would not be able to electrostatically hinder the chloride ion flow. On the other, the ligand also has a lower number of interactions with the lining pore residues (either hydrogen bond interactions or hydrophobic ones), indicating weaker binding. Hence, we will focus on the trans-Azo-NZ1 docking poses (Fig. 7B) where the sulfonate group is placed in the narrower 2' pore region (down), as it seems to be the most likely blocking site for trans-Azo-NZ1. Such a ligand orientation is stabilized by multiple hydrogen bond interactions of the amide group of the nitrazepam core of Azo-NZ1 with the hydroxyl group of one of the five T13' residues, as well as with the backbone of T10' residues. Further stabilization is provided by the hydrophobic contacts involving the apolar region of the nitrazepam core, the side chain of L9' and the methyl group of T6' (Fig. 7B). The length of the ligand (14.1 $\AA$ ) is optimal to place the polar amide group close to the polar T13' side chain and, most importantly, the negatively charged sulfonate group is placed in the more constricted region of the pore, the 2' ring, since T13' and A2' are $\sim 16 \AA$ apart. Thus, the hydrogen bond seen with the 2' residue in $\mathrm{GABA}_{A}$ and GABA rho2 receptors (Maleeva et al., 2019) is important for orienting the ligand with the sulfonate toward the extracellular part of the receptor, but it is not essential for inhibition, as it can be seen with these newest results on GlyRs. Indeed, sulfonate binding at the 2' 


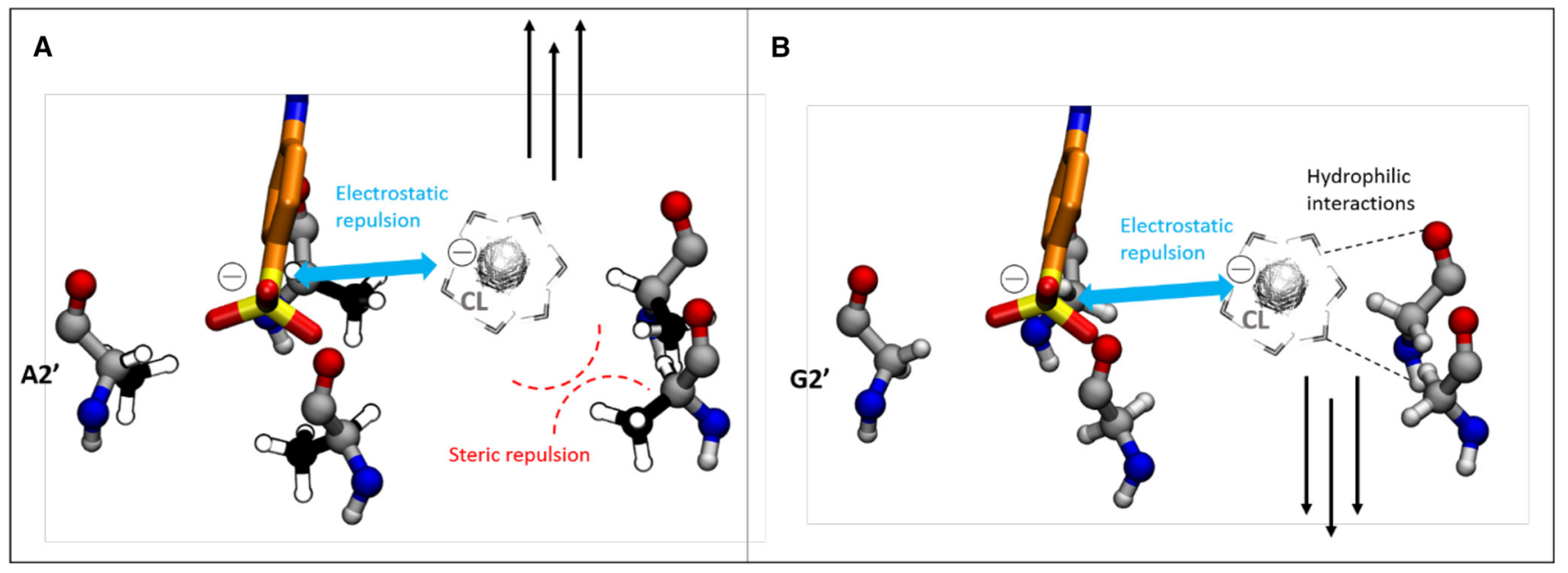

\section{a1G254A GlyR}

a1 GlyR

Figure 8. Proposed scheme of the passage of a hydrated chloride ion through the 2' pore region in $\alpha 1$ G254A and $\alpha 1$ GlyRs. The hydrated chloride ion is represented in greyscale. Electrostatic repulsion between the negatively charged sulfonate group and the chloride ion is represented with a blue arrow, the steric hindrance between the chloride ion and the methyl side chain of A2' is shown as curved red dashed lines, and the hydrophilic interactions between the water molecules in the chloride ion solvation shell and the G2' backbone are represented as black dashed lines. $\boldsymbol{A}$, In the $\alpha 1$ G254A GlyR mutant, the electrostatic and steric barrier created by the combined effect of the sulfonate group of trans-Azo-NZ1 and A2', respectively, does not let the chloride ion go through this narrow part of the pore. $\boldsymbol{B}$, In wild-type $\alpha 1 \mathrm{GlyR}$, the electrostatic repulsion with the sulfonate group is not enough to prevent chloride conduction, as the residue at the 2' position is now glycine, so that the pore has more space and the chloride ion can also form hydrophilic interactions with the G2' backbone.

position is possible as long as the 2' ring has the right diameter to accommodate the sulfonate group. In this regard, the volume of A2' in $\alpha 1 \mathrm{G} 254 \mathrm{~A}$ GlyR is similar to that of S2' in GABA rho2 and the $\gamma$ subunit of GABA recep- $^{2}$ res tors. Moreover, the interactions with other pore-lining residues (6', 9', and 13') appear to have a stronger contribution to the binding of trans-Azo-NZ1 in GlyRs than in GABARs.

The only difference between $\alpha 1$ and $\alpha 2$ Gly receptors in the pore channel is the change from glycine to alanine. This minor change produces different inhibitory activities for both $\alpha$ GlyRs, mainly decreasing the inhibitory effect of transAzo-NZ1 for $\alpha 1$ GlyR. The Ala2' residue in $\alpha 2$ GlyR is placing five methyl groups, one from each of the five M2 helixes, in the narrowest part of the pore, compared with five hydrogen atoms for the Gly2' ring in $\alpha 1$ GlyR. This decreases the diameter of the pore at the 2' position by at least $1 \AA$ in $\alpha 2$ GlyR, and thus makes it easier for the sulfonate group to clog the pore. Interestingly, the negatively charged sulfonate group of trans-Azo-NZ1 (down pose) occupies the same position as the chloride ion trapped in x-ray structures of GABA $_{A} R$ (Laverty et al., 2017) and GlyR (Huang et al., 2017). Thus, Ala2' residues add steric hindrance (Fig. $8 A$ ), which would be another obstacle for the hydrated chloride ion, besides the electrostatic repulsion with the sulfonate group of trans-Azo-NZ1. In contrast, Gly2' loses this steric repulsion (Fig. 8B) and potentially adds a favorable ion-dipole interaction with chloride, since its backbone is more exposed into the pore (Sunesen et al., 2006).

The results for the heteromeric $\alpha 2 / \beta$ GlyR are similar to the G254A $\alpha 1$ mutant GlyR, for both trans- and cis-Azo-
NZ1 conformers (Extended Data Figs. 7-3, 7-6). In particular, the down binding pose of trans-Azo-NZ1 is almost identical for $\alpha 1 \mathrm{G} 254 \mathrm{~A}$ and $\alpha 2 / \beta$ GlyRs (Extended Data Fig. 7-3B,C), in agreement with the experimental observation of both receptors being inhibited by the trans isomer. In other words, although the replacement of two Ala residues in the 2' ring ( $\alpha 1$ G254A and $\alpha 2)$ by Pro $(\beta)$ reduces the diameter of the pore at this position by $\sim 2 \AA$, it still allows sulfonate binding.

The docking results obtained rationalize the stronger blocking effect of trans-Azo-NZ1 on $\alpha 2$ GlyRs compared with $\alpha 1$ GlyR and the relief of the pore blocking-mediated inhibition of $\alpha 2$ GlyRs on cis isomerization. However, $\alpha 1$ GlyRs show an unexpected behavior, in that they are weakly inhibited by trans-Azo-NZ1 but inhibition increases slightly on UV irradiation (Fig. 2). Considering our docking results for cis-Azo-NZ1 inside the pore (Extended Data Figs. 7-4A, 7-5A) and the lack of inhibition of $\alpha 1$ GlyR at saturating glycine concentrations (Fig. 2B), we explored other possible interaction sites of cis-Azo-NZ1 outside the M2 pore. Our results (Extended Data Fig. 7-7) suggest that trans-Azo-NZ1 binds preferentially inside the pore (Extended Data Fig. 7-7C,D), whereas cis-Azo-NZ1 might bind in both the $\mathrm{M} 2$ pore-lining region and an alternative site at the interface between the extracellular domain (ECD) and TMD (Extended Data Fig. 7-7A,B), analogously to another azo-nitrazepam-based GlyR inhibitor, Glyght (Gomila et al., 2020). Moreover, cis-Azo-NZ1 has a slightly higher probability to interact with ECD-TMD region (35\%) than the pore (28\%) in $\alpha 1$ GlyR, whereas in $\alpha 2$ GlyR it mostly binds in the pore (46\%). Altogether, we 
surmise that cis-Azo-NZ1 might be able to inhibit $\alpha 1$ GlyR by binding to the ECD-TMD interface.

\section{Discussion}

This study presents the experimental and modeling analysis of the action of Azo-NZ1 on $\alpha 1$ and $\alpha 2$ subunits of GlyR heterologously expressed in cultured cells and also on glycinergic synaptic currents in whole-cell recordings from hypoglossal motoneurons of brainstem slices. Our observations demonstrate that Azo-NZ1 is an efficient and subunit-specific photoswitchable inhibitor of GlyRs with the following main differences between its action on $\alpha 1$ and $\alpha 2$ GlyR subunits: (1) in trans-configuration, Azo-NZ1 caused strong inhibition of glycine-induced currents on cells expressing homomeric $\alpha 2$ GlyRs, while it was not able to modulate $\alpha 1$ GlyRs responses at saturating concentrations of glycine; (2) in cis-configuration, induced by UV illumination, Azo-NZ1 completely lost the ability to inhibit $\alpha 2$ GlyRs.

The aforementioned differences in the interaction of Azo-NZ1 with $\alpha 1$ and $\alpha 2$ GlyRs, as well as the fact that the sensitivity of $\alpha 2$ GlyRs to Azo-NZ1 $\left(\mathrm{IC}_{50}=9 \mu \mathrm{M}\right)$ is much higher than the one of GlyR $\alpha 1$ and $\mathrm{GABA}_{A} \mathrm{Rs}$ $\left(I C_{50}=67 \mu \mathrm{m}\right.$; Maleeva et al., 2019), suggest that this photochromic compound might be used as selective light-dependent modulator of $\alpha 2$-containing GlyRs.

Extrasynaptic homomeric GlyR $\alpha 2$ or GlyR $\alpha 3$ were detected in the striatum, hippocampus, and prefrontal cortex (McCracken et al., 2017), and in neurons of the trigeminal mesencephalic nucleus (Bae et al., 2018). Tonically active GlyRs also regulate the firing of medium spiny neurons of the dorsal striatum and may thus affect the function of basal ganglia (Molchanova et al., 2018). As the TM2 domains, forming the ion selective pore is identical for GlyR $\alpha 2$ and GlyR $\alpha 3$, these extrasynaptic receptors tonically regulating firing properties of neuronal circuits represent the important target for Azo-NZ1 action.

The properties of Azo-NZ1 suggest that this compound may represent a promising tool for the pharmacological discrimination of the extrasynaptic GlyRs and for the studying of the processes mediated by them, as well as for light-controlled regulation of neuronal plasticity and excitability in the different brain regions.

The features of Azo-NZ1 action indicate that Azo-NZ1 interacts primarily with the ion pore domain of $\alpha 2$ GlyRs. This assumption is supported by our experiments demonstrating that substitution of single amino acid (G254A) situated in 2' position of the pore-forming TM2 domain of $\alpha 1$ receptors was sufficient to switch the mode of Azo-NZ1 action, which became similar to those observed for $\alpha 2$ GlyRs (Fig. 6B,C). An additional argument for the pore action of this photoswitch is provided by the comparison of Azo-NZ1 effects on homomeric and heteromeric GlyRs formed by $\alpha 2$ or $\alpha 2 / \beta$ subunits. Incorporation of $\beta$ subunits, which possess proline at the 2' position, caused a decrease in the blocking ability of Azo-NZ1 without changing the light-controlled switching (Fig. $3 F, G$ ). This is consistent with our recent observations on homomeric rho1 $\mathrm{GABA}_{C}$ receptors, which demonstrated that proline in 2' position of TM2 results in the complete absence of Azo-NZ1 inhibitory effect (Maleeva et al., 2019).

The modeling of Azo-NZ1 molecular interactions with GlyR suggests that the pore blocking effect of trans-AzoNZ1 is because of two essential features of the photochromic molecule. First, the negative charge of the sulfonate group, which mimics that of the chloride ion. Second, its longitude (14.1 $\AA$ ) which allows Azo-NZ1 to form hydrogen bonds between the amide group of the nitrazepam core and T13' and place the sulfonate in the 2' region, thus spanning the whole pore (Fig. $7 B$ ). In the 2' ring, the sulfonate group is able to (sterically and electrostatically) block chloride conduction depending on the 2' residue, glycine (in $\alpha 1$ subunits) or alanine (in $\alpha 2$ subunits). With glycine, the hydrated chloride is still able to go through the 2' ring thanks to the exposed hydrophilic backbone of glycine and the wider space in that constriction (Fig. 8B). In contrast, alanine has a methyl group that increases steric hindrance (together with the steric and electrostatic barrier of the sulfonate group, see Fig. $8 A$ ), resulting in the strong inhibition of this type of GlyR.

Differences in the gating of $\alpha 1$ and $\alpha 2$ GlyRs may play as well an important role in the interaction of Azo-NZ1 with $\alpha 1$ and $\alpha 2$ GlyRs, along with differences in the pore geometry. In our single-channel recordings, $\mathrm{NP}_{\mathrm{o}}$ for $\alpha 2$ GlyRs in control is drastically higher than $\mathrm{NP}_{\mathrm{o}}$ for $\alpha 1$ receptors. This is in accordance with previous works demonstrating that the mean open time of $\alpha 2$ channels is almost 100 -fold longer than the one of $\alpha 1$ (Takahashi et al., 1992). Accordingly, Azo-NZ1 interaction with the channel pore of $\alpha 1$ GlyRs might be additionally prevented by a short channel openings characteristic of this receptor.

Several previously described inhibitors of GlyRs were shown to act through the pore of the channel. CTB blocks $\alpha 1$ GlyRs with high efficiency, while being two orders magnitude less efficient at $\alpha 2$ GlyRs (Rundström et al., 1994). Exchange of the TM2 domain of the $\alpha 1$ subunit by the one of $\alpha 2$ imparted resistance to CTB to the receptor, indicating that the negatively charged CTB acts as poreblocker. Also, the uncharged GlyR inhibitor PTX was shown to interact with the pore of GlyRs (Zhorov and Bregestovski, 2000; Yang et al., 2007; Gielen et al., 2015; Gielen and Corringer, 2018). Its blocking ability at homomeric $\alpha 1$ and $\alpha 2$ GlyRs was similar, however, incorporation of $\beta$ subunit caused a drastic decrease in PTX blocking activity on heteromeric GlyRs (Pribilla et al., 1992). It was suggested that both 6' and 2' amino acids of the pore are involved in binding of PTX (Yang et al., 2007).

Another inhibitor of GlyRs, NFA, was shown to block the $\mathrm{Cl}$-selective ion channel (Maleeva et al., 2017). NFA has $\sim 10$-fold higher affinity to $\alpha 2$ than to $\alpha 1$ GlyRs and G254A mutation in $\alpha 1$ receptors increases its sensitivity to NFA, indicating the Ala2' residue in TM2 pore domain as the main site of its action. However, the $\beta$ subunit does not have a strong impact on the interaction of NFA with GlyRs (Maleeva et al., 2017). Therefore, the 2' amino acids ring of the TM2 pore is the key point of action of different channel blockers. Azo-NZ1 has a distinct profile of interaction with GlyRs. Similarly to NFA, it inhibits much 
stronger $\alpha 2$ receptors than $\alpha 1$ GlyRs, while, similarly to PTX, incorporation of $\beta$ subunit decreased sensitivity of $\alpha 2$ receptors to Azo-NZ1. These similarities support common interaction points inside of the channel pore that Azo-NZ1 shares with PTX and NFA.

Analysis of single-channels recordings of $\alpha 2$ GlyRs activity on application of Azo-NZ1 disclosed another very interesting aspect of their interaction. UV illumination at high $(50 \mu \mathrm{M})$ concentration of Azo-NZ1 produced a strong transient increase of the open probability of $\alpha 2$ channels. Apparently Azo-NZ1 while being switched from trans- to cis-states is able to induce elevation of the open probability of $\alpha 2$ channels. This effect was completely absent at application of Azo-NZ1 to $\alpha 1$ GlyRs, confirming the mismatch of their interaction sites. The molecular mechanism of this transient potentiation of $\alpha 2$ GlyRs needs to be clarified.

Our electrophysiological analysis in the mouse hypoglossal motoneuron, proved Azo-NZ1 to be a photoswitchable modulator of the glycinergic elPSCs and further support the suggestion that the photochrome is a selective modulator of synaptic transmission mediated by $\alpha 2$ GlyRs. We show that at the high concentrations causing complete block of $\alpha 2$-containing GlyRs (Fig. 3D), trans-Azo-NZ1 inhibited the amplitude of glycinergic elPSCs only by $\sim 50 \%$ (Fig. $5 C, D$ ). This is in line with earlier observations demonstrating that during the early postnatal development, the subunit composition of GlyRs in the hypoglossal motoneurons continuously changes, resulting in the decreasing of "fetal" $\alpha 2$ subunits and increasing of "adult" $\alpha 1$ subunits expression (Malosio et al., 1991; Singer et al., 1998). At the intermediate age (P5-P8) of the mice we used in the present study, the motoneurons express nearly equal proportion of $\alpha 1$ and $\alpha 2$ GlyR subunits (Singer et al., 1998). It is also well documented that the peak concentration of glycine acting on postsynaptic receptors of motoneurons is $\sim 3 \mathrm{~mm}$ (Beato, 2008). At these highly saturating agonist concentrations, $\alpha 1$ GlyRs are not inhibited by trans-Azo-NZ1 (Fig. 2B) and the photochrome should operate as specific blocker of $\alpha 2$ GlyRs and thus fetal glycinergic synapses. Another reason for the moderate blocking of elPSCs is that they are mainly mediated by synaptic $\alpha 2 / \beta$ receptors, which, as we have demonstrated in heterologous expression system, are less sensitive to Azo-NZ1 inhibitory effect. Future studies should be aimed at investigating the effect of AzoNZ1 on the tonic currents that are mediated by homomeric receptors, as Azo-NZ1 demonstrate highest affinity for $\alpha 2$ homomeric receptors and thus can reveal itself to be an excellent tool for unravelling their physiological role.

In conclusion, we present here the detailed analysis of the novel photochromic compound Azo-NZ1 action on different GlyRs expressed heterologously and on synaptic glycinergic currents in brain slices. Experimental observations, supported by modeling analysis, demonstrate that Azo-NZ1 is a potent TM2 pore blocker of $\alpha 2$ GlyRs. This subunit-specific light-controlled modulator of GlyRs may be a useful tool for controlling neuronal activity in spinal cord, brainstem, cerebellum and other parts of nervous system with high expression of glycinergic synapses.

\section{References}

Akagi H, Miledi R (1988) Heterogeneity of glycine receptors and their messenger RNAs in rat brain and spinal cord. Science 242:270273.

Bae JY, Lee JS, Ko SJ, Cho YS, Rah J-C, Cho HJ, Park MJ, Bae YC (2018) Extrasynaptic homomeric glycine receptors in neurons of the rat trigeminal mesencephalic nucleus. Brain Struct Funct 223:2259-2268.

Banghart M, Borges K, Isacoff E, Trauner D, Kramer RH (2004) Lightactivated ion channels for remote control of neuronal firing. Nat Neurosci 7:1381-1386.

Banghart MR, Mourot A, Fortin DL, Yao JZ, Kramer RH, Trauner D (2009) Photochromic blockers of voltage-gated potassium channels. Angew Chem Int Ed Engl 48:9097-9101.

Bartels E, Wassermann NH, Erlanger BF (1971) Photochromic activators of the acetylcholine receptor. Proc Natl Acad Sci USA 68:1820-1823.

Beato M (2008) The time course of transmitter at glycinergic synapses onto motoneurons. J Neurosci 28:7412-7425.

Becker CM, Hoch W, Betz H (1988) Glycine receptor heterogeneity in rat spinal cord during postnatal development. EMBO $\mathrm{J}$ 7:37173726.

Berlin S, Szobota S, Reiner A, Carroll EC, Kienzler MA, Guyon A, Xiao T, Trauner D, Isacoff EY (2016) A family of photoswitchable NMDA receptors. Elife 5:e12040.

Broichhagen J, Frank JA, Trauner D (2015) A roadmap to success in photopharmacology. Acc Chem Res 48:1947-1960.

Chattipakorn SC, McMahon LL (2002) Pharmacological characterization of glycine-gated chloride currents recorded in rat hippocampal slices. J Neurophysiol 87:1515-1525.

Cohen J, Arkhipov A, Braun R, Schulten K (2006) Imaging the migration pathways for $\mathrm{O} 2, \mathrm{CO}, \mathrm{NO}$, and $\mathrm{Xe}$ inside myoglobin. Biophys $\mathrm{J}$ 91:1844-1857.

Colquhoun D, Sigworth FJ (1995) Fitting and statistical analysis of single-channel records. In: Single-channel recording, pp 483-587. Boston: Springer.

David-Watine B, Goblet C, de Saint Jan D, Fucile S, Devignot V, Bregestovski P, Korn H (1999) Cloning, expression and electrophysiological characterization of glycine receptor alpha subunit from zebrafish. Neuroscience 90:303-317.

Du J, Lü W, Wu S, Cheng Y, Gouaux E (2015) Glycine receptor mechanism elucidated by electron cryo-microscopy. Nature 526:224229.

Durrant JD, McCammon JA (2011) BINANA: a novel algorithm for ligand-binding characterization. J Mol Graph Model 29:888-893.

Fehrentz T, Huber FME, Hartrampf N, Bruegmann T, Frank JA, Fine NHF, Malan D, Danzl JG, Tikhonov DB, Sumser M, Sasse P, Hodson DJ, Zhorov BS, Klöcker N, Trauner D (2018) Optical control of L-type $\mathrm{Ca} 2+$ channels using a diltiazem photoswitch. Nat Chem Biol 14:764-767.

Fucile S, de Saint Jan D, David-Watine B, Korn H, Bregestovski P (1999) Comparison of glycine and GABA actions on the zebrafish homomeric glycine receptor. J Physiol 517:369-383.

Gielen M, Corringer PJ (2018) The dual-gate model for pentameric ligand-gated ion channels activation and desensitization. J Physiol 596:1873-1902.

Gielen M, Thomas P, Smart TG (2015) The desensitization gate of inhibitory Cys-loop receptors. Nat Commun 6:6829.

Gomila AMJ, Rustler K, Maleeva G, Nin-Hill A, Wutz D, BautistaBarrufet A, Rovira X, Bosch M, Mukhametova E, Petukhova E, Ponomareva D, Mukhamedyarov M, Peiretti F, Alfonso-Prieto M, Rovira C, König B, Bregestovski P, Gorostiza P (2020) Photocontrol of endogenous glycine receptors in vivo. Cell Chem Biol 21:2451-2456.

Gorostiza P, Isacoff E (2007) Optical switches and triggers for the manipulation of ion channels and pores. Mol Biosyst 3:686-704.

Gorostiza P, Isacoff EY (2008) Optical switches for remote and noninvasive control of cell signaling. Science 322:395-399. 
Hanwell MD, Curtis DE, Lonie DC, Vandermeersch T, Zurek E, Hutchison GR (2012) Avogadro: an advanced semantic chemical editor, visualization, and analysis platform. J Cheminform 4:17.

Harvey AJ, Abell AD (2001) Alpha-ketoester-based photobiological switches: synthesis, peptide chain extension and assay against alpha-chymotrypsin. Bioorg Med Chem Lett 11:2441-2444.

Harvey RJ, Depner UB, Wässle H, Ahmadi S, Heindl C, Reinold H, Smart TG, Harvey K, Schütz B, Abo-Salem OM, Zimmer A, Poisbeau P, Welzl H, Wolfer DP, Betz H, Zeilhofer HU, Müller U (2004) GlyR a3: an essential target for spinal PGE2-mediated inflammatory pain sensitization. Science 304:884-887. doi:10.1126/ science.1094925

Haverkamp S, Müller U, Harvey K, Harvey RJ, Betz H, Wässle H (2003) Diversity of glycine receptors in the mouse retina: localization of the alpha3 subunit. J Comp Neurol 465:524-539.

Hawthorne R, Cromer BA, Ng HL, Parker MW, Lynch JW (2006) Molecular determinants of ginkgolide binding in the glycine receptor pore. J Neurochem 98:395-407.

Huang X, Shaffer PL, Ayube S, Bregman H, Chen H, Lehto SG, Luther JA, Matson DJ, McDonough SI, Michelsen K, Plant MH, Schneider S, Simard JR, Teffera Y, Yi S, Zhang M, DiMauro EF, Gingras J (2017) Crystal structures of human glycine receptor $\alpha 3$ bound to a novel class of analgesic potentiators. Nat Struct Mol Biol 24:108-133.

Huckvale R, Mortensen M, Pryde D, Smart TG, Baker JR (2016) Azogabazine: a photochromic antagonist of the GABAA receptor. Org Biomol Chem 14:6676-6678.

Humphrey W, Dalke A, Schulten K (1996) VMD: visual molecular dynamics. J Mol Graph 14:33-38.

Kienzler MA, Reiner A, Trautman E, Yoo S, Trauner D, Isacoff EY (2013) A red-shifted, fast-relaxing azobenzene photoswitch for visible light control of an ionotropic glutamate receptor. J Am Chem Soc 135:17683-17686.

Kondratskaya EL, Betz H, Krishtal OA, Laube B (2005) The $\beta$ subunit increases the ginkgolide $B$ sensitivity of inhibitory glycine receptors. Neuropharmacology 49:945-951.

Laverty D, Thomas P, Field M, Andersen OJ, Gold MG, Biggin PC, Gielen M, Smart TG (2017) Crystal structures of a GABAA-receptor chimera reveal new endogenous neurosteroid-binding sites. Nat Struct Mol Biol 24:977-985.

Lester HA, Krouse ME, Nass MM, Wassermann NH, Erlanger BF (1979) Light-activated drug confirms a mechanism of ion channel blockade. Nature 280:509-510.

Lin WC, Tsai MC, Davenport CM, Smith CM, Veit J, Wilson NM, Adesnik H, Kramer RH (2015) A comprehensive optogenetic pharmacology toolkit for in vivo control of GABA(A) receptors and synaptic inhibition. Neuron 88:879-891.

Low SE, Ito D, Hirata H (2018) Characterization of the zebrafish glycine receptor family reveals insights into glycine receptor structure function and stoichiometry. Front Mol Neurosci 11:286.

Lynch JW, Zhang Y, Talwar S, Estrada-Mondragon A (2017) Glycine receptor drug discovery. Adv Pharmacol 79:225-253.

Maleeva G, Buldakova S, Bregestovski P (2015) Selective potentiation of alpha 1 glycine receptors by ginkgolic acid. Front Mol Neurosci 8:64.

Maleeva G, Peiretti F, Zhorov BS, Bregestovski P (2017) Voltage-dependent inhibition of glycine receptor channels by niflumic acid. Front Mol Neurosci 10:125.

Maleeva G, Wutz D, Rustler K, Nin-Hill A, Rovira C, Petukhova E, Bautista -Barrufet A, Gomila-Juaneda A, Scholze P, Peiretti F, Alfonso-Prieto M, König B, Gorostiza P, Bregestovski P (2019) A photoswitchable GABA receptor channel blocker. Br J Pharmacol 176:2661-2677.

Malosio ML, Marquèze -Pouey B, Kuhse J, Betz H (1991) Widespread expression of glycine receptor subunit mRNAs in the adult and developing rat brain. EMBO J 10:2401-2409.

McCracken LM, Lowes DC, Salling MC, Carreau-Vollmer C, Odean NN, Blednov YA, Betz H, Harris RA, Harrison NL (2017) Glycine receptor $\alpha 3$ and $\alpha 2$ subunits mediate tonic and exogenous agonist- induced currents in forebrain. Proc Natl Acad Sci USA 114:E7179E7186.

McCool BA, Botting SK (2000) Characterization of strychnine-sensitive glycine receptors in acutely isolated adult rat basolateral amygdala neurons. Brain Res 859:341-351.

Meyer G, Kirsch J, Betz H, Langosch D (1995) Identification of a gephyrin binding motif on the glycine receptor beta subunit. Neuron 15:563-572.

Molchanova SM, Comhair J, Karadurmus D, Piccart E, Harvey RJ, Rigo J-M, Schiffmann SN, Brône B, Gall D (2018) Tonically active $\alpha 2$ subunit-containing glycine receptors regulate the excitability of striatal medium spiny neurons. Front Mol Neurosci 10:442.

Mukhtarov M, Ragozzino D, Bregestovski P (2005) Dual Ca2 + modulation of glycinergic synaptic currents in rodent hypoglossal motoneurones. J Physiol 569:817-831.

Mukhtarov M, Liguori L, Waseem T, Rocca F, Buldakova S, Arosio D, Bregestovski P (2013) Calibration and functional analysis of three genetically encoded $\mathrm{Cl}(-) / \mathrm{pH}$ sensors. Front Mol Neurosci 6:9.

Petukhova E, Ponomareva D, Mukhamedyarov M, Maleeva G, Bregestovski $P$ (2018) Developmental changes in the inhibition of glycinergic synaptic currents by niflumic acid in hypoglossal motoneurons. Front Mol Neurosci 11:416.

Pettersen EF, Goddard TD, Huang CC, Couch GS, Greenblatt DM, Meng EC, Ferrin TE (2004) UCSF chimera - a visualization system for exploratory research and analysis. J Comput Chem 25:16051612.

Pribilla I, Takagi T, Langosch D, Bormann J, Betz H (1992) The atypical M2 segment of the beta subunit confers picrotoxinin resistance to inhibitory glycine receptor channels. EMBO J 11:4305-4311.

Raju SG, Barber AF, LeBard DN, Klein ML, Carnevale V (2013) Exploring volatile general anesthetic binding to a closed membrane-bound bacterial voltage-gated sodium channel via computation. PLoS Comput Biol 9:e1003090-10.

Richter L, de Graaf C, Sieghart W, Varagic Z, Mörzinger M, de Esch IJP, Ecker GF, Ernst M (2012) Diazepam-bound GABAA receptor models identify new benzodiazepine binding-site ligands. Nat Chem Biol 8:455-464.

Rundström N, Schmieden V, Betz H, Bormann J, Langosch D (1994) Cyanotriphenylborate: subtype-specific blocker of glycine receptor chloride channels. Proc Natl Acad Sci USA 91:8950-8954.

Sassoe-Pognetto M, Wassle H, Grunert U (1994) Glycinergic synapses in the rod pathway of the rat retina: cone bipolar cells express the alpha 1 subunit of the glycine receptor. J Neurosci 14:51315146.

Schmid K, Böhmer G, Gebauer K (1991) Glycine receptor-mediated fast synaptic inhibition in the brainstem respiratory system. Respir Physiol 84:351-361.

Singer JH, Talley EM, Bayliss DA, Berger AJ (1998) Development of glycinergic synaptic transmission to rat brain stem motoneurons. $J$ Neurophysiol 80:2608-2620.

Sunesen M, de Carvalho LP, Dufresne V, Grailhe R, Savatier-Duclert N, Gibor G, Peretz A, Attali B, Changeux J-P, Paas Y (2006) Mechanism of $\mathrm{Cl}$-selection by a glutamate-gated chloride (GluCl) receptor revealed through mutations in the selectivity filter. J Biol Chem 281:14875-14881.

Takahashi T, Momiyama A, Hirai K, Hishinuma F, Akagi H (1992) Functional correlation of fetal and adult forms of glycine receptors with developmental changes in inhibitory synaptic receptor channels. Neuron 9:1155-1161.

Tochitsky I, Banghart MR, Mourot A, Yao JZ, Gaub B, Kramer RH, Trauner D (2012) Optochemical control of genetically engineered neuronal nicotinic acetylcholine receptors. Nat Chem 4:105-111.

Trott O, Olson AJ (2010) AutoDock Vina: improving the speed and accuracy of docking with a new scoring function, efficient optimization, and multithreading. J Comput Chem 31:455-461.

Vafa B, Lewis TM, Cunningham AM, Jacques P, Lynch JW, Schofield PR (1999) Identification of a new ligand binding domain in the alpha1 subunit of the inhibitory glycine receptor. J Neurochem 73:2158-2166. 
Vandenberg RJ, French CR, Barry PH, Shine J, Schofield PR (1992) Antagonism of ligand-gated ion channel receptors: two domains of the glycine receptor alpha subunit form the strychnine-binding site. Proc Natl Acad Sci USA 89:1765-1769.

Volgraf M, Gorostiza P, Numano R, Kramer RH, Isacoff EY, Trauner D (2006) Allosteric control of an ionotropic glutamate receptor with an optical switch. Nat Chem Biol 2:47-52.

Wang P, Slaughter M (2005) Effects of GABA receptor antagonists on retinal glycine receptors and on homomeric glycine receptor alpha subunits. J Neurophysiol 93:3120-3126.

Waterhouse A, Bertoni M, Bienert S, Studer G, Tauriello G, Gumienny R, Heer FT, de Beer TAP, Rempfer C, Bordoli L, Lepore R, Schwede T (2018) SWISS-MODEL: homology modelling of protein structures and complexes. Nucleic Acids Res 46:W296W303.
Yang Z, Cromer BA, Harvey RJ, Parker MW, Lynch JW (2007) A proposed structural basis for picrotoxinin and picrotin binding in the glycine receptor pore. J Neurochem 103:580-589.

Young AB, Snyder SH (1973) Strychnine binding associated with glycine receptors of the central nervous system. Proc Natl Acad Sci USA 70:2832-2836.

Zarbin MA, Wamsley JK, Kuhar MJ (1981) Glycine receptor: light microscopic autoradiographic localization with $[3 \mathrm{H}]$ strychnine. J Neurosci 1:532-547.

Zeilhofer HU, Acuña MA, Gingras J, Yévenes GE (2018) Glycine receptors and glycine transporters: targets for novel analgesics? Cell Mol Life Sci 75:447-465.

Zhorov BS, Bregestovski PD (2000) Chloride channels of glycine and GABA receptors with blockers: Monte Carlo minimization and structure-activity relationships. Biophys J 78:1786-1803. 